\title{
BIMODULE MONOMORPHISM CATEGORIES AND RSS EQUIVALENCES VIA COTILTING MODULES
}

\author{
BAO-LIN XIONG PU ZHANG* YUE-HUI ZHANG
}

\begin{abstract}
The monomorphism category $\mathscr{S}(A, M, B)$ induced by a bimodule ${ }_{A} M_{B}$ is the subcategory of $\Lambda$-mod consisting of $\left[\begin{array}{c}X \\ Y\end{array}\right]_{\phi}$ such that $\phi: M \otimes_{B} Y \rightarrow X$ is a monic $A$-map, where $\Lambda=\left[\begin{array}{cc}A & M \\ 0 & B\end{array}\right]$. In general, it is not the monomorphism categories induced by quivers. It could describe the Gorenstein-projective $\Lambda$ modules. This monomorphism category is a resolving subcategory of $\Lambda$-mod if and only if $M_{B}$ is projective. In this case, it has enough injective objects and Auslander-Reiten sequences, and can be also described as the left perpendicular category of a unique basic cotilting $\Lambda$-module. If $M$ satisfies the condition (IP), then the stable category of $\mathscr{S}(A, M, B)$ admits a recollement of additive categories, which is in fact a recollement of singularity categories if $\mathscr{S}(A, M, B)$ is a Frobenius category. Ringel-Schmidmeier-Simson equivalence between $\mathscr{S}(A, M, B)$ and its dual is introduced. If $M$ is an exchangeable bimodule, then an RSS equivalence is given by a $\Lambda$ - $\Lambda$ bimodule which is a two-sided cotilting $\Lambda$-module with a special property; and the Nakayama functor $\mathcal{N}_{\Lambda}$ gives an RSS equivalence if and only if both $A$ and $B$ are Frobenius algebras.

Keywords: monomorphism category induced by bimodule, Auslander-Reiten sequence, cotilting module, recollement of additive categories, exchangeable bimodule, RSS equivalence, Frobenius algebra, Nakayama functor
\end{abstract}

\section{Introduction and preliminaries}

1.1. Throughout, algebras mean Artin algebras, modules are finitely generated, and a subcategory is a full subcategory closed under isomorphisms. For an algebra $A$, let $A$-mod $(\operatorname{resp} . \bmod A)$ be the category of left (resp. right) $A$-modules. So there is a duality $\mathrm{D}: A-\bmod \rightarrow \bmod A$.

This paper is to draw attention to the monomorphism category $\mathscr{S}(A, M, B)$ induced by an $A$ - $B$-bimodule $M$. It is defined to be the subcategory of $\Lambda$-mod consisting of left $\Lambda$-modules $[\underset{Y}{X}]_{\phi}$ such that $\phi: M \otimes_{B}$ $Y \rightarrow X$ is a monic $A$-map, where $\Lambda$ is the triangular matrix algebra $\left[\begin{array}{cc}A & M \\ 0 & B\end{array}\right]$. When ${ }_{A} M_{B}={ }_{A} A_{A}$, it is the classical submodule category $\mathscr{S}(A)$ in [RS1-RS3]. This submodule category is initiated in [Bir]. C. Ringel and M. Schmidmeier [RS2] establish its Auslander-Reiten theory; and D. Simson ([S1]-[S3]) studies its representation type. By D. Kussin, H. Lenzing and H. Meltzer ([KLM1, KLM2]; see also [C]), it is related to the singularity theory. It has been generalized via quivers to the filtered chain category and the separated monomorphism category ([S1-S3], [Z1], [LZ], [ZX]). However, all these generalizations can not include monomorphism categories induced by bimodules (this will be clarified in Example [5.8). Another motivation is that $\mathscr{S}(A, M, B)$ can describe the Gorenstein-projective $\Lambda$-modules ([Z2, Thms. 1.4., 2.2]).

1.2. To study $\mathscr{S}(A, M, B)$, first, we need it to be a resolving subcategory of $\Lambda$-mod. So we work under the condition that $M_{B}$ is projective: this is a necessary and sufficient condition such that $\mathscr{S}(A, M, B)$ is a resolving subcategory. Then $\mathscr{S}(A, M, B)$ has enough projective objects and enough injective objects, and it

Supported by the NSF of China (11431010, 11301019, 11271251).

2010 Mathematics Subject Classification. Primary 16G70; Secondary 16D40; 16E30

*The corresponding author. 
is a Frobenius category if and only if $A$ and $B$ are selfinjective and ${ }_{A} M$ and $M_{B}$ are projective (Corollary 2.3). This monomorphism category $\mathscr{S}(A, M, B)$ enjoys the functorially finiteness and Auslander-Reiten sequences, and it closely relates to the tilting theory. Here we use the classical cotilting modules of injective dimension at most 1 ([HR], [R, p.167], [AR], [ASS, p.242]). For a left $\Lambda$-module $Z$, let ${ }^{\perp} Z$ denote the subcategory $\left\{L \in \Lambda-\bmod \mid \operatorname{Ext}_{\Lambda}^{m}(L, Z)=0, \forall m \geq 1\right\}$.

Theorem 1.1. Let $M$ be an A-B-bimodule. Then

(1) The following are equivalent:

(i) $M_{B}$ is projective;

(ii) $\mathscr{S}(A, M, B)$ is a resolving subcategory of $\Lambda$-mod;

(iii) ${ }_{\Lambda} T:=\left[\begin{array}{c}\mathrm{D}\left(A_{A}\right) \\ 0\end{array}\right] \oplus\left[\begin{array}{c}E_{\mathrm{D}\left(B_{B}\right)} \\ \mathrm{D}\left(B_{B}\right)\end{array}\right]_{e}$ is a unique cotilting left $\Lambda$-module, up to multiplicities of indecomposable direct summands, such that $\mathscr{S}(A, M, B)={ }^{\perp} T$, where $E_{\mathrm{D}\left(B_{B}\right)}$ is an injective envelope of the left $A$-module ${ }_{A} M \otimes_{B} \mathrm{D}\left(B_{B}\right)$ with inclusion e $: M \otimes_{B} \mathrm{D}\left(B_{B}\right) \hookrightarrow E_{\mathrm{D}\left(B_{B}\right)}$.

(2) $\mathscr{S}(A, M, B)$ is a contravariantly finite subcategory of $\Lambda$-mod. Moreover, if $M_{B}$ is projective, then $\mathscr{S}(A, M, B)$ is a functorially finite subcategory of $\Lambda$-mod, and has Auslander-Reiten sequences.

Corollary 1.2. If ${ }_{A} M_{B}$ satisfies the condition (IP), then ${ }_{\Lambda} T=\left[\begin{array}{c}\mathrm{D}\left(A_{A}\right) \\ 0\end{array}\right] \oplus\left[\begin{array}{c}M \otimes_{B} \mathrm{D}(B) \\ \mathrm{D}\left(B_{B}\right)\end{array}\right]_{\mathrm{Id}}$ is a unique cotilting left $\Lambda$-module, up to multiplicities of indecomposable direct summands, such that $\mathscr{S}(A, M, B)={ }^{\perp} T$.

1.3. A recollement is first introduced for triangulated categories ([BBD]), and then for abelian categories ([MV], $[\mathrm{PS}],[\mathrm{Ku}]$ ). It becomes a powerful tool in triangulated categories and in abelian categories (see e.g. [Kö], [H2], [IKM], [FP], [PV], [FZ]). One can also consider recollements of additive categories in the similar way. For a subcategory $\mathscr{X}$ of an additive category $\mathscr{A}$, recall that the objects of the stable category $\mathscr{A} / \mathscr{X}$ are the objects of $\mathscr{A}$, and $\operatorname{Hom}_{\mathscr{A} / \mathscr{X}}(M, N):=\operatorname{Hom}_{\mathscr{A}}(M, N) /(M, \mathscr{X}, N)$, where $(M, \mathscr{X}, N)$ is the subgroup consisting of those morphisms factoring through objects of $\mathcal{X}$. For an algebra $A$, denote $A$-mod $/ \operatorname{inj}(A)$ by $A$ - $\overline{\bmod }$, where $\operatorname{inj}(A)$ is the subcategory of the injective $A$-modules. Similarly, $\overline{\mathscr{S}(A, M, B)}$ is the stable category of $\mathscr{S}(A, M, B)$ respect to the subcategory of the injective objects of $\mathscr{S}(A, M, B)$.

Theorem 1.3. An A-B-bimodule satisfying the condition (IP) induces a recollement of additive categories

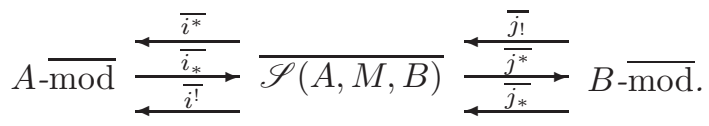

If in addition $A$ and $B$ are selfinjective algebras, then it is in fact a recollement of singularity categories.

Here the singularity category $\mathcal{D}_{s g}^{b}(\Lambda)$ of an algebra $\Lambda$ is defined to be the Verdier quotient $\mathcal{D}_{s g}^{b}(\Lambda):=$ $\mathcal{D}^{b}(\Lambda$-mod $) / K^{b}(\operatorname{proj}(\Lambda))$, where $\mathcal{D}^{b}(\Lambda$-mod $)$ is the bounded derived category, and $K^{b}(\operatorname{proj}(\Lambda))$ is the bounded homotopy category. See R. Buchweitz [Buch] and D. Orlov [O].

1.4. The dual of $\mathscr{S}(A, M, B)$ is the epimorphism category $\mathscr{F}(A, M, B)$. The right module version of $\mathscr{S}(A, M, B)$ is $\mathscr{S}(A, M, B)_{r}$, and $\mathscr{S}(A, M, B)_{r}$ is a resolving subcategory if and only if ${ }_{A} M$ is projective; in this case, there is a unique basic cotilting right $\Lambda$-module $U$ such that $\mathscr{S}(A, M, B)_{r}={ }^{\perp}\left(U_{\Lambda}\right)$. Then $\mathscr{F}(A, M, B)$ can be also described as D $\mathscr{S}(A, M, B)_{r}$. Ringel-Schmidmeier-Simson equivalence $\mathscr{S}(A, M, B) \cong$ 
$\mathscr{F}(A, M, B)$ is studied. Such an equivalence implies a strong symmetry, and was first observed by C. Ringel and M. Schidmeier [RS2] for the case of ${ }_{A} M_{B}={ }_{A} A_{A}$, and by D. Simson [S1] for a chain without relations, and then developed to acyclic quivers with monomial relations in [ZX].

We introduce exchangeable bimodules. If ${ }_{A} M_{B}$ is exchangeable, then the unique left cotilting $\Lambda$-module $T$ with $\mathscr{S}(A, M, B)={ }^{\perp} T$ (cf. Corollary 1.2) can be endowed with a $\Lambda$ - $\Lambda$-bimodule structure via the exchangeable bimodule isomorphism, such that the right module $T_{\Lambda}$ coincides with the unique right cotilting $\Lambda$-module $U$ with $\mathscr{S}(A, M, B)_{r}={ }^{\perp}\left(U_{\Lambda}\right)$. This two-sided cotilting $\Lambda$-module ${ }_{\Lambda} T_{\Lambda}$ enjoys a good property in the sense that $\operatorname{End}_{\Lambda}\left({ }_{\Lambda} T\right)^{o p} \cong \Lambda$ as algebras, and under this isomorphism of algebras, $T_{\operatorname{End}_{\Lambda}\left({ }_{\Lambda} T\right)^{o p} \text { coincides }}$ with $T_{\Lambda}$. These good properties of $T$ induce an RSS equivalence:

Theorem 1.4. Let ${ }_{A} M_{B}$ be an exchangeable bimodule. Then $T=\left[\begin{array}{c}\mathrm{D}(A) \\ 0\end{array}\right] \oplus\left[\begin{array}{c}M \otimes_{B} \mathrm{D}(B) \\ \mathrm{D}(B)\end{array}\right]_{\mathrm{Id}}$ can be endowed with a $\Lambda$ - $\Lambda$-bimodule such that $\mathrm{D} \mathrm{Hom}_{\Lambda}(-, T): \mathscr{S}(A, M, B) \cong \mathscr{F}(A, M, B)$ is an RSS equivalence.

The Nakayama functor $\mathcal{N}_{\Lambda}$ gives an RSS equivalence if and only if both $A$ and $B$ are Frobenius algebras (Proposition [5.5). Examples show that if ${ }_{A} M_{B}$ is not exchangeable, then an RSS equivalence can not be guaranteed. Examples also show that the monomorphism category $\mathscr{S}(A, M, B)$ is not the separated monomorphism category of the corresponding quiver in the sense of [ZX], in general (see Example 5.8). However, we do not know a sufficient and necessary condition and the uniqueness of an RSS equivalence. See Subsection 5.5.

1.5. Let $M$ be an $A$-B-bimodule. The multiplication of the associated matrix algebra $\Lambda=\left[\begin{array}{cc}A & M \\ 0\end{array}\right]$ is given by $\left[\begin{array}{cc}a & m \\ 0 & b\end{array}\right]\left[\begin{array}{cc}a^{\prime} & m^{\prime} \\ 0 & b^{\prime}\end{array}\right]=\left[\begin{array}{cc}a a^{\prime} & a m^{\prime}+m b^{\prime} \\ 0 & b b^{\prime}\end{array}\right]$. Each left $\Lambda$-module is identified with a triple $\left[\begin{array}{l}X \\ Y\end{array}\right]_{\phi}$, where $X \in A$-mod, $Y \in B$ mod, and $\phi: M \otimes_{B} Y \rightarrow X$ is an $A$-map; and a $\Lambda$-map is identified with a pair $\left[\begin{array}{l}f_{1} \\ f_{2}\end{array}\right]:\left[\begin{array}{l}X_{1} \\ Y_{1}\end{array}\right]_{\phi_{1}} \rightarrow\left[\begin{array}{l}X_{2} \\ Y_{2}\end{array}\right]_{\phi_{2}}$, where $f_{1}: X_{1} \rightarrow X_{2}$ is an $A$-map, and $f_{2}: Y_{1} \rightarrow Y_{2}$ a $B$-map, such that the diagram

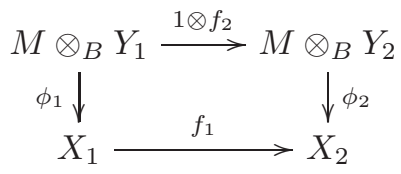

commutes. Under this identification, the indecomposable projective $\Lambda$-modules are exactly $\left[\begin{array}{c}P \\ 0\end{array}\right]$ and $\left[\begin{array}{c}M \otimes_{B} Q \\ Q\end{array}\right]_{\mathrm{Id}}$ ' where $P$ and $Q$ run over the indecomposable projective $A$-modules and $B$-modules, respectively. The indecomposable injective $\Lambda$-modules are $\left[\operatorname{Hom}_{A}^{I}(M, I)\right]_{\varphi}$ and $\left[\begin{array}{l}0 \\ J\end{array}\right]$, where $I$ and $J$ run over the indecomposable injective $A$-modules and $B$-modules, respectively ([ARS, p.73]). Throughout, for any left $A$-module $X$, we denote by $\varphi=\varphi_{X}$ the left $A$-map $M \otimes_{B} \operatorname{Hom}_{A}(M, X) \longrightarrow X$ given by $\varphi(m \otimes f)=f(m)$, i.e., the adjunction isomorphism $\operatorname{Hom}_{A}\left(M \otimes_{B} \operatorname{Hom}_{A}(M, X), X\right) \cong \operatorname{Hom}_{B}\left(\operatorname{Hom}_{A}(M, X), \operatorname{Hom}_{A}(M, X)\right)$ sends $\varphi$ to $\operatorname{Id}_{\operatorname{Hom}_{A}(M, X)}$. We will call $\varphi$ the involution map.

1.6. Conditions on a bimodule. A bimodule ${ }_{A} M_{B}$ satisfies the condition (IP), if $M \otimes_{B} \mathrm{D}\left(B_{B}\right)$ is an injective left $A$-module and $M_{B}$ is projective.

A bimodule ${ }_{A} M_{B}$ is exchangeable, if both ${ }_{A} M$ and $M_{B}$ are projective and there is an $A$ - $B$-bimodule isomorphism $\mathrm{D}\left({ }_{A} A_{A}\right) \otimes_{A} M \cong M \otimes_{B} \mathrm{D}\left({ }_{B} B_{B}\right)$, which is called an exchangeable bimodule isomorphism. 
By adjunction isomorphisms we have $A$ - $B$-bimodule isomorphisms

$$
\mathrm{D}\left(\mathrm{D} A \otimes_{A} M\right) \cong \operatorname{Hom}_{A}(M, A), \quad \mathrm{D}\left(M \otimes_{B} \mathrm{D} B\right) \cong \operatorname{Hom}_{B}(M, B) .
$$

Thus ${ }_{A} M_{B}$ is exchangeable if and only if there is an $A$ - $B$-bimodule isomorphism $\operatorname{Hom}_{A}(M, A) \cong \operatorname{Hom}_{B}(M, B)$; and if and only if there is an $A$-B-bimodule isomorphism $\mathcal{N}_{A}\left({ }_{A} M\right) \cong \mathcal{N}_{B}\left(M_{B}\right)$, where $\mathcal{N}_{A}$ denotes the Nakayama functor $\mathrm{D}_{\operatorname{Hom}}(-, A)$.

For ${ }_{A} N$, let $\operatorname{add}(N)$ be the subcategory of $A$-mod of direct summands of finite direct sums of $N$.

Example 1.5. (1) An exchangeable bimodule ${ }_{A} M_{B}$ satisfies the condition (IP), and $\mathrm{D}\left({ }_{A} A\right) \otimes_{A} M$ is an injective right $B$-module.

In fact, since ${ }_{A} M$ is projective, $\mathrm{D}\left({ }_{A} A_{A}\right) \otimes_{A} M \in \operatorname{add}\left(\mathrm{D}\left({ }_{A} A_{A}\right) \otimes_{A} A\right)=\operatorname{add}\left(\mathrm{D}\left(A_{A}\right)\right)$, so $\mathrm{D}\left({ }_{A} A_{A}\right) \otimes_{A} M$ is an injective left $A$-module. Thus $M \otimes_{B} \mathrm{D}\left({ }_{B} B_{B}\right) \cong \mathrm{D}\left({ }_{A} A_{A}\right) \otimes_{A} M$ is an injective left A-module. Similarly, one can prove that $\mathrm{D}\left({ }_{A} A\right) \otimes_{A} M$ is an injective right $B$-module.

(2) If $B=A$ and $M=A \oplus \cdots \oplus A$, then ${ }_{A} M_{A}$ is an exchangeable bimodule.

(3) For an algebra $A$, let $B:=A \oplus \cdots \oplus A$, and ${ }_{A} M_{B}:={ }_{A} B_{B}$. Then ${ }_{A} M_{B}$ is an exchangeable bimodule.

(4) For an algebra $B$, let $A:=B \oplus \cdots \oplus B$, and ${ }_{A} M_{B}:={ }_{A} A_{B}$. Then ${ }_{A} M_{B}$ is an exchangeable bimodule.

(5) An algebra $A$ over field $k$ is symmetric, if $\mathrm{D}\left({ }_{A} A_{A}\right) \cong{ }_{A} A_{A}$ as $A$-A-bimodules. If both $A$ and $B$ are symmetric algebras and $M={ }_{A} P \otimes_{k} Q_{B}$, where ${ }_{A} P$ and $Q_{B}$ are projective, then ${ }_{A} M_{B}$ is an exchangeable bimodule.

(6) An algebra $B$ is Frobenius, if $\mathrm{D}\left(B_{B}\right) \cong{ }_{B} B$ as left $B$-modules. If $B$ is a Frobenius algebra and ${ }_{A} M_{B}$ is a bimodule with ${ }_{A} M$ injective and $M_{B}$ projective, then ${ }_{A} M_{B}$ satisfies the condition (IP).

(7) Let $B$ be a selfinjective algebra, and ${ }_{A} M_{B}$ a bimodule with $M_{B}$ projective. Then ${ }_{A} M_{B}$ satisfies the condition (IP) if and only if ${ }_{A} M$ is injective. In particular, if both $A$ and $B$ are selfinjective $k$-algebras and $M={ }_{A} P \otimes_{k} Q_{B}$, where ${ }_{A} P$ and $Q_{B}$ are projective, then ${ }_{A} M_{B}$ satisfies the condition (IP).

In fact, since $B$ is a selfinjective algebra, $\mathrm{D}\left(B_{B}\right) \in \operatorname{add}\left({ }_{B} B\right)$, and hence ${ }_{A} M \otimes_{B} \mathrm{D}\left(B_{B}\right) \in \operatorname{add}\left({ }_{A} M\right)$. So $M \otimes_{B} \mathrm{D}\left(B_{B}\right)$ is an injective $A$-module if and only if ${ }_{A} M$ is injective.

\section{Monomorphism categories induced by bimodules}

2.1. Recall that the monomorphism category $\mathscr{S}(A, M, B)$ induced by bimodule ${ }_{A} M_{B}$ is the subcategory of $\Lambda$-mod consisting of $\left[\begin{array}{l}X \\ Y\end{array}\right]_{\phi}$ such that $\phi: M \otimes_{B} Y \longrightarrow X$ is a monic $A$-map. So it contains all the projective $\Lambda$-modules and is closed under direct sums and direct summands.

Lemma 2.1. Let ${ }_{A} M_{B}$ be a bimodule. Then $\mathscr{S}(A, M, B)$ is closed under extensions. Thus $\mathscr{S}(A, M, B)$ is an exact category with the canonical exact structure, and hence a Krull-Schmidt category. 
Proof. For an exact sequence $0 \rightarrow\left[\begin{array}{l}X_{1} \\ Y_{1}\end{array}\right]_{\phi_{1}} \stackrel{\left[\begin{array}{l}f_{1} \\ g_{1}\end{array}\right]}{\longrightarrow}\left[\begin{array}{l}X \\ Y\end{array}\right]_{\phi} \stackrel{\left[\begin{array}{l}f_{2} \\ g_{2}\end{array}\right]}{\longrightarrow}\left[\begin{array}{l}X_{2} \\ Y_{2}\end{array}\right]_{\phi_{2}} \rightarrow 0$ in $\Lambda$-mod with $\left[\begin{array}{l}X_{1} \\ Y_{1}\end{array}\right]_{\phi_{1}} \in \mathscr{S}(A, M, B)$ and $\left[\begin{array}{l}X_{2} \\ Y_{2}\end{array}\right]_{\phi_{2}} \in \mathscr{S}(A, M, B)$, we get a commutative diagram

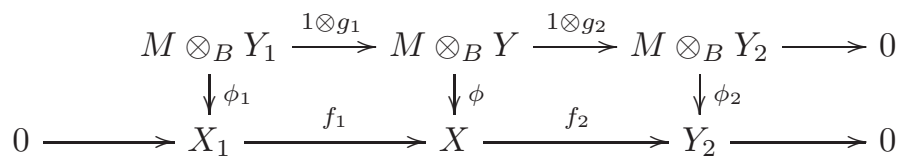

with exact rows. It follows from the Snake Lemma that $\phi$ is monic, i.e., $[\underset{Y}{X}]_{\phi} \in \mathscr{S}(A, M, B)$.

Proposition 2.2. Let ${ }_{A} M_{B}$ be a bimodule such that $M_{B}$ is projective. Then

(1) $\mathscr{S}(A, M, B)$ has enough projective objects; and the indecomposable projective objects of $\mathscr{S}(A, M, B)$ are exactly the indecomposable projective $\Lambda$-modules.

(2) $\mathscr{S}(A, M, B)$ has enough injective objects; and the indecomposable injective objects are exactly $\left[\begin{array}{l}I \\ 0\end{array}\right]$ and $\left[\begin{array}{c}E_{J} \\ J\end{array}\right]_{e}$, where $I$ (resp. $J$ ) runs over indecomposable injective left A-modules (resp. B-modules), and $E_{J}$ is an injective envelope of the left $A$-module $M \otimes_{B} J$ with inclusion $e: M \otimes_{B} J \hookrightarrow E_{J}$.

In particular, if $M$ satisfies the condition (IP), then the indecomposable injective objects of $\mathscr{S}(A, M, B)$ are exactly $\left[\begin{array}{l}I \\ 0\end{array}\right]$ and $\left[\underset{J}{M \otimes_{B} J}\right]_{\mathrm{Id}}$.

Proof. (1) Projective $\Lambda$-modules are clearly projective objects of $\mathscr{S}(A, M, B)$. For any object $\left[\begin{array}{l}X \\ Y\end{array}\right]_{\phi} \in$ $\mathscr{S}(A, M, B)$, taknig projective covers $\pi_{Y}:{ }_{B} Q \rightarrow{ }_{B} Y$ and $\pi_{C}:{ }_{A} P \rightarrow{ }_{A}$ Coker $(\phi)$, we get exact sequences $0 \rightarrow \operatorname{Ker}\left(\pi_{Y}\right) \stackrel{i_{Y}}{\rightarrow} Q \stackrel{\pi_{Y}}{\rightarrow} Y \rightarrow 0$ and $0 \rightarrow \operatorname{Ker}\left(\pi_{C}\right) \stackrel{i_{G}}{\rightarrow} P \stackrel{\pi_{G}}{\rightarrow} \operatorname{Coker}(\phi) \rightarrow 0$. Consider the exact sequence $0 \longrightarrow M \otimes_{B} Y \stackrel{\phi}{\longrightarrow} X \stackrel{\pi}{\longrightarrow} \operatorname{Coker}(\phi) \longrightarrow 0$. We get an $A$-map $\theta: P \rightarrow X$ such that $\pi_{C}=\pi \theta$, and hence the commutative diagram in $A$-mod with exact rows

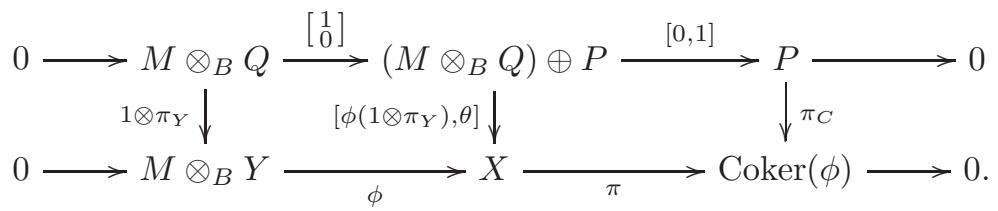

Since $M_{B}$ is projective, $0 \longrightarrow M \otimes_{B} \operatorname{Ker}\left(\pi_{Y}\right) \stackrel{1 \otimes i_{Y}}{\longrightarrow} M \otimes_{B} Q \stackrel{1 \otimes \pi_{Y}}{\longrightarrow} M \otimes_{B} Y \longrightarrow 0$ is an exact sequence of $A$-module. By the Snake Lemma we get the commutative diagram in $A$-mod with exact rows and columns

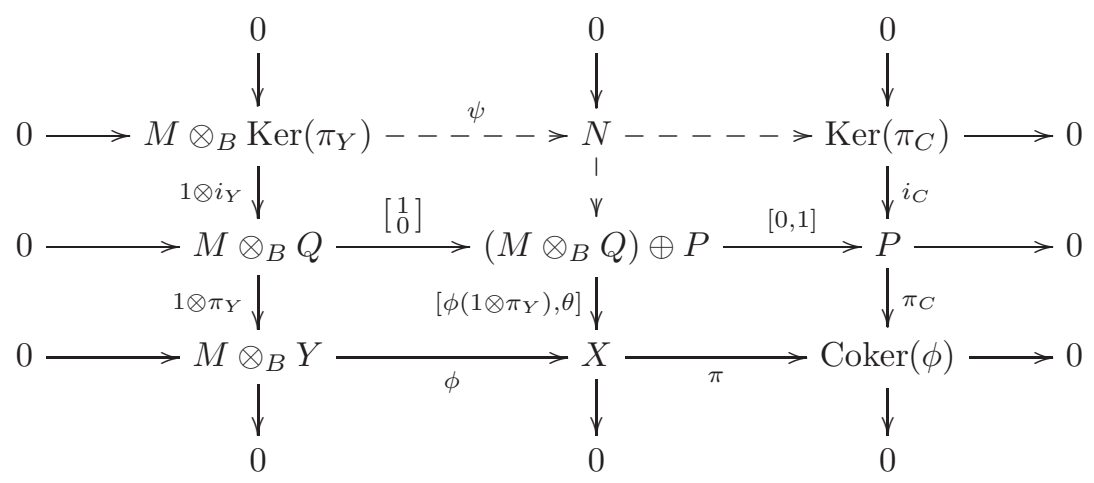


So the left and middle columns give the exact sequence

$$
0 \longrightarrow\left[\underset{\operatorname{Ker}\left(\pi_{Y}\right)}{N}\right]_{\psi} \longrightarrow\left[\begin{array}{l}
P \\
0
\end{array}\right] \oplus\left[\begin{array}{c}
M \otimes_{B} Q \\
Q
\end{array}\right]_{\mathrm{Id}} \longrightarrow\left[\begin{array}{l}
X \\
Y
\end{array}\right]_{\phi} \longrightarrow 0
$$

in $\mathscr{S}(A, M, B)$. This shows that $\mathscr{S}(A, M, B)$ has enough projective objects.

Let $\left[\begin{array}{l}X \\ Y\end{array}\right]_{\phi}$ be an indecomposable projective object of $\mathscr{S}(A, M, B)$. Then the exact sequence $(*)$ splits and $\left[\begin{array}{l}X \\ Y\end{array}\right]_{\phi}$ is a direct summand of $\left[\begin{array}{c}P \\ 0\end{array}\right] \oplus\left[\begin{array}{c}M \otimes_{B} Q \\ Q\end{array}\right]_{\mathrm{Id}}$. By Lemma 2.1, $\mathscr{S}(A, M, B)$ is a Krull-Schimdt category, so $\left[\begin{array}{c}X \\ Y\end{array}\right]_{\phi}$ is isomorphic to $\left[\begin{array}{c}P^{\prime} \\ 0\end{array}\right]$ or $\left[\begin{array}{c}M \otimes_{B} Q^{\prime} \\ Q^{\prime}\end{array}\right]_{\mathrm{Id}}$, where $P^{\prime}$ (resp. $\left.Q^{\prime}\right)$ is an indecomposable projective $A$-module (resp. $B$-module). Thus $[\underset{Y}{X}]_{\phi}$ is a projective $\Lambda$-module.

(2) It is clear that $\left[\begin{array}{l}I \\ 0\end{array}\right]$ and $\left[\begin{array}{c}E_{J} \\ J\end{array}\right]_{e}$ are indecomposable objects of $\mathscr{S}(A, M, B)$. We show that they are injective objects of $\mathscr{S}(A, M, B)$. Put $\left[\begin{array}{c}W \\ V\end{array}\right]_{\phi}$ to be $\left[\begin{array}{c}I \\ 0\end{array}\right]$ or $\left[\begin{array}{c}E_{J} \\ J\end{array}\right]_{e}$. For an exact sequence in $\mathscr{S}(A, M, B)$

$$
0 \longrightarrow\left[\begin{array}{l}
X_{1} \\
Y_{1}
\end{array}\right]_{\phi_{1}} \stackrel{\left[\begin{array}{c}
f_{1} \\
g_{1}
\end{array}\right]}{\longrightarrow}\left[\begin{array}{c}
X_{2} \\
Y_{2}
\end{array}\right]_{\phi_{2}} \stackrel{\left[\begin{array}{l}
f_{2} \\
g_{2}
\end{array}\right]}{\longrightarrow}\left[\begin{array}{c}
X_{3} \\
Y_{3}
\end{array}\right]_{\phi_{3}} \longrightarrow 0
$$

with $\left[\begin{array}{l}\alpha \\ \beta\end{array}\right] \in \operatorname{Hom}_{\Lambda}\left(\left[\begin{array}{l}X_{1} \\ Y_{1}\end{array}\right]_{\phi_{1}},\left[\begin{array}{l}W \\ V\end{array}\right]_{\phi}\right)$, we need looking for $\left[\begin{array}{l}\gamma \\ \delta\end{array}\right] \in \operatorname{Hom}_{\Lambda}\left(\left[\begin{array}{l}X_{2} \\ Y_{2}\end{array}\right]_{\phi_{2}},\left[\begin{array}{l}W \\ V\end{array}\right]_{\phi}\right)$ such that $\left[\begin{array}{l}\alpha \\ \beta\end{array}\right]=\left[\begin{array}{l}\gamma \\ \delta\end{array}\right]\left[\begin{array}{l}f_{1} \\ g_{1}\end{array}\right]$. Since ${ }_{B} V$ is an injective module and $g_{1}: Y_{1} \rightarrow Y_{2}$ is monic, there is a $B$-map $\delta: Y_{2} \rightarrow V$ such that $\beta=\delta g_{1}$. Consider the $A$-map $\phi(1 \otimes \delta): M \otimes_{B} Y_{2} \rightarrow W$. Since ${ }_{A} W$ is an injective module and $\phi_{2}: M \otimes_{B} Y_{2} \rightarrow X_{2}$ is monic, there is an $A$-map $\gamma^{\prime}: X_{2} \rightarrow W$ such that $\phi(1 \otimes \delta)=\gamma^{\prime} \phi_{2}$. Since $\alpha \phi_{1}=\phi(1 \otimes \beta)$ and $f_{1} \phi_{1}=\phi_{2}\left(1 \otimes g_{1}\right)$, we have

$$
\alpha \phi_{1}=\phi(1 \otimes \beta)=\phi(1 \otimes \delta)\left(1 \otimes g_{1}\right)=\gamma^{\prime} \phi_{2}\left(1 \otimes g_{1}\right)=\gamma^{\prime} f_{1} \phi_{1} .
$$

So $\alpha-\gamma^{\prime} f_{1}=\eta \pi_{1}$ for some $\eta: \operatorname{Coker}\left(\phi_{1}\right) \rightarrow W$. Since $h_{1}: \operatorname{Coker}\left(\phi_{1}\right) \rightarrow \operatorname{Coker}\left(\phi_{2}\right)$ is monic and $W$ is injective, there is an $A$-map $\eta^{\prime}: \operatorname{Coker}\left(\phi_{2}\right) \rightarrow W$ such that $\eta=\eta^{\prime} h_{1}$. We present the process as the diagram with exact rows and columns:

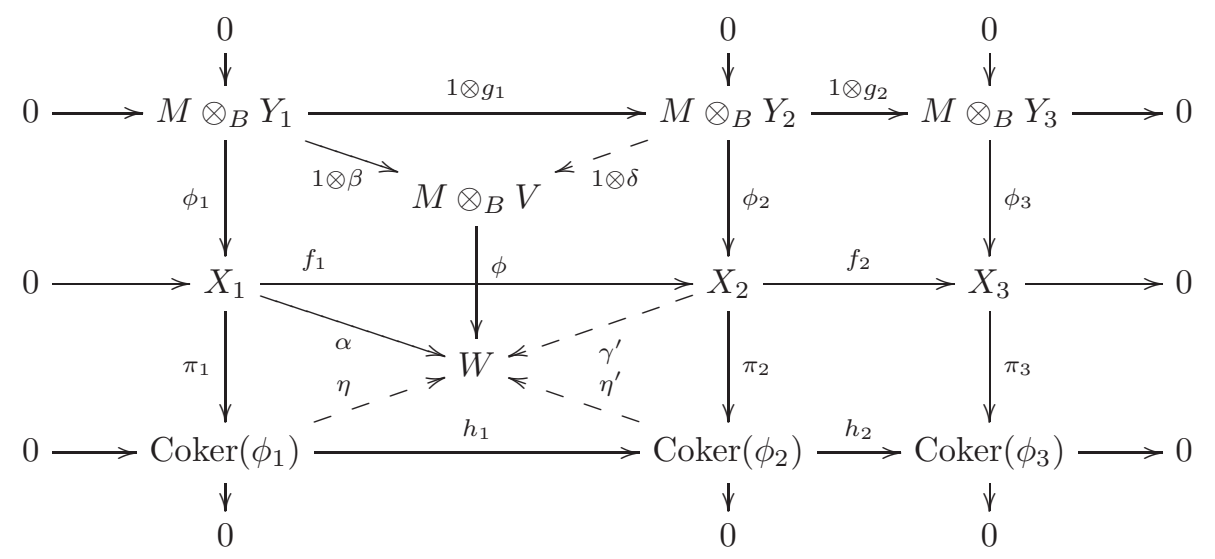

Put $\gamma:=\gamma^{\prime}+\eta^{\prime} \pi_{2} \in \operatorname{Hom}_{A}\left(X_{2}, W\right)$. Then

$$
\gamma \phi_{2}=\left(\gamma^{\prime}+\eta^{\prime} \pi_{2}\right) \phi_{2}=\gamma^{\prime} \phi_{2}=\phi(1 \otimes \delta)
$$

and

$$
\gamma f_{1}=\left(\gamma^{\prime}+\eta^{\prime} \pi_{2}\right) f_{1}=\gamma^{\prime} f_{1}+\eta^{\prime} \pi_{2} f_{1}=\gamma^{\prime} f_{1}+\eta^{\prime} h_{1} \pi_{1}=\gamma^{\prime} f_{1}+\eta \pi_{1}=\alpha .
$$

This shows $\left[\begin{array}{l}\gamma \\ \delta\end{array}\right] \in \operatorname{Hom}_{\Lambda}\left(\left[\begin{array}{l}X_{2} \\ Y_{2}\end{array}\right]_{\phi_{2}},\left[\begin{array}{l}W \\ V\end{array}\right]_{\phi}\right)$ and $\left[\begin{array}{l}\alpha \\ \beta\end{array}\right]=\left[\begin{array}{l}\gamma \\ \delta\end{array}\right]\left[\begin{array}{l}f_{1} \\ g_{1}\end{array}\right]$. 
Next, we show that $\mathscr{S}(A, M, B)$ has enough injective objects. For $\left[{ }_{Y}^{X}\right]_{\phi} \in \mathscr{S}(A, M, B)$, taking injective envelopes $\iota_{Y}: Y \rightarrow J$ and $\iota_{C}: \operatorname{Coker}(\phi) \rightarrow I$, we get exact sequences $0 \rightarrow Y \stackrel{\iota_{Y}}{\rightarrow} J \stackrel{p_{Y}}{\rightarrow} \operatorname{Coker}\left(\iota_{Y}\right) \rightarrow 0$ and $0 \rightarrow \operatorname{Coker}(\phi) \stackrel{\iota_{G}}{\rightarrow} I \stackrel{p_{C}}{\rightarrow} \operatorname{Coker}\left(\iota_{C}\right) \rightarrow 0$. We take an injective envelope of the left $A$-module $M \otimes_{B} J$ with inclusion $e: M \otimes_{B} J \rightarrow E_{J}$. Since $\phi: M \otimes_{B} Y \rightarrow X$ is monic and $E_{J}$ is an injective module, there is an $A$-map $\alpha: X \rightarrow E_{J}$ satisfying $\alpha \phi=e\left(1 \otimes \iota_{Y}\right)$, and hence we get a commutative diagram with exact rows:

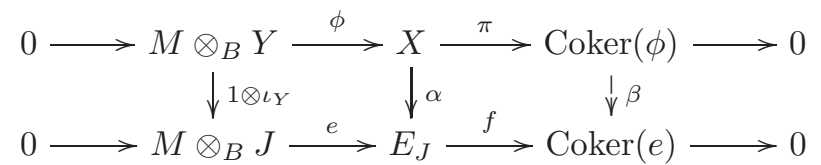

Since $M_{B}$ is projective, we have a commutative diagram with exact rows and columns:

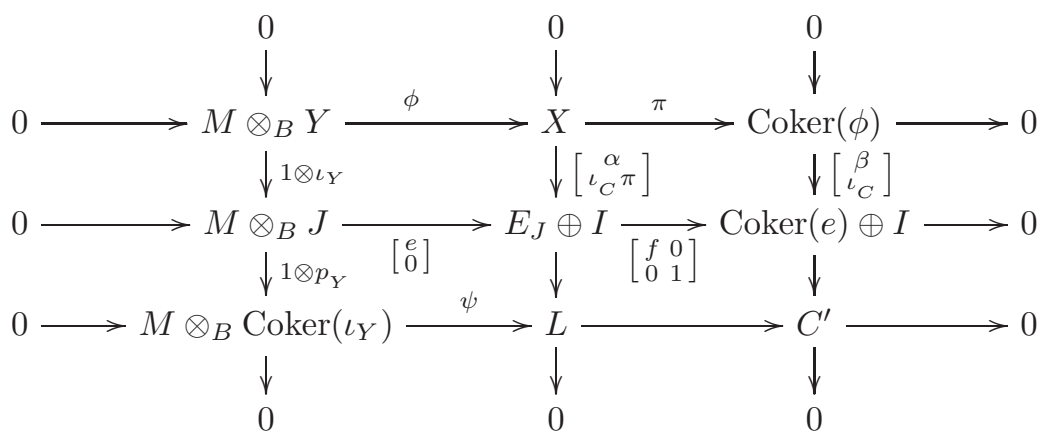

(since $\iota_{C}$ is monic, applying the Snake Lemma to the upper two rows we see that $\psi$ is monic). The left and middle columns give a short exact sequence in $\mathscr{S}(A, M, B)$

$$
0 \longrightarrow\left[\begin{array}{l}
X \\
Y
\end{array}\right]_{\phi} \longrightarrow\left[\begin{array}{l}
I \\
0
\end{array}\right] \oplus\left[\begin{array}{c}
E_{J} \\
J
\end{array}\right]_{e} \longrightarrow\left[\underset{\operatorname{Coker}\left(\iota_{Y}\right)}{L}\right]_{\psi} \longrightarrow 0
$$

This shows that $\mathscr{S}(A, M, B)$ has enough injective objects.

Finally, if $\left[\begin{array}{l}X \\ Y\end{array}\right]_{\phi}$ is an indecomposable injective object of $\mathscr{S}(A, M, B)$, then $(* *)$ splits. By Lemma 2.1 , $\mathscr{S}(A, M, B)$ is a Krull-Schimdt category. So $\left[\begin{array}{c}X \\ Y\end{array}\right]_{\phi}$ is either of the form $\left[\begin{array}{c}I \\ 0\end{array}\right]$ or of the form $\left[\begin{array}{c}E_{J} \\ J\end{array}\right]_{e}$.

Corollary 2.3. Let ${ }_{A} M_{B}$ be a bimodule with $M_{B}$ projective. Then $\mathscr{S}(A, M, B)$ is a Frobenius category (with the canonical exact structure) if and only if both $A$ and $B$ are selfinjective algebras and ${ }_{A} M$ is projective.

Proof. By Proposition 2.2, $\mathscr{S}(A, M, B)$ is a Frobenius category if and only if

$$
\operatorname{add}\left(\left[\begin{array}{l}
A \\
0
\end{array}\right] \oplus\left[\begin{array}{l}
M \\
B
\end{array}\right]_{\mathrm{Id}}\right)=\operatorname{add}\left(\left[\begin{array}{c}
\mathrm{D}\left(A_{A}\right) \\
0
\end{array}\right] \oplus\left[\begin{array}{c}
E_{\mathrm{D}\left(B_{B}\right)} \\
\mathrm{D}\left(B_{B}\right)
\end{array}\right]_{e}\right),
$$

where $E_{\mathrm{D}\left(B_{B}\right)}$ is an injective envelope of the $A$-module $M \otimes_{B} \mathrm{D}\left(B_{B}\right)$ with embedding $e: M \otimes_{B} \mathrm{D}\left(B_{B}\right) \rightarrow$ $E_{\mathrm{D}\left(B_{B}\right)}$. Thus, if $\mathscr{S}(A, M, B)$ is Frobenius, then ${ }_{A} A$ is injective (thus $\left.\operatorname{add}\left(\mathrm{D}\left(A_{A}\right)\right)=\operatorname{add}\left({ }_{A} A\right)\right),{ }_{A} M$ is injective (thus ${ }_{A} M \in \operatorname{add}\left(\mathrm{D}\left(A_{A}\right)\right)=\operatorname{add}\left({ }_{A} A\right)$, hence ${ }_{A} M$ is projective), and ${ }_{B} B$ is injective. Conversely, if $A$ and $B$ are selfinjective and ${ }_{A} M$ is projective, then $\operatorname{add}\left({ }_{B} B\right) \cong \operatorname{add}\left(\mathrm{D}\left(B_{B}\right)\right)$, hence $M \otimes_{B} \mathrm{D}\left(B_{B}\right) \in$ $\operatorname{add}\left(M \otimes_{B} B\right)=\operatorname{add}\left({ }_{A} M\right)$, so $M \otimes_{B} \mathrm{D}\left(B_{B}\right)$ is a projective left $A$-module, and hence an injective left $A$ module. Thus $M \otimes_{B} \mathrm{D}\left(B_{B}\right)=E_{\mathrm{D}\left(B_{B}\right)}$. So $\operatorname{add}\left(\left[\begin{array}{c}E_{\mathrm{D}\left(B_{B}\right)} \\ \mathrm{D}\left(B_{B}\right)\end{array}\right]_{e}\right)=\operatorname{add}\left(\left[\begin{array}{c}M \otimes_{B} \mathrm{D}\left(B_{B}\right) \\ \mathrm{D}\left(B_{B}\right)\end{array}\right]_{\mathrm{Id}}\right)=\operatorname{add}\left(\left[\begin{array}{c}M \\ B\end{array}\right]_{\mathrm{Id}}\right)$, thus $\operatorname{add}\left(\left[\begin{array}{c}A \\ 0\end{array}\right] \oplus\left[\begin{array}{c}M \\ B\end{array}\right]_{\mathrm{Id}}\right)=\operatorname{add}\left(\left[\begin{array}{c}\mathrm{D}\left(A_{A}\right) \\ 0\end{array}\right] \oplus\left[\begin{array}{c}E_{\mathrm{D}\left(B_{B}\right)} \\ \mathrm{D}\left(B_{B}\right)\end{array}\right]_{e}\right)$. Hence $\mathscr{S}(A, M, B)$ is a Frobenius category. 
2.2. To prove Theorem 1.1 we need some preparations. A subcategory is a resolving subcategory of $\Lambda$-mod, if it contains all the projective $\Lambda$-modules and is closed under extensions, kernels of epimorphisms and direct summands $([\mathrm{AR}])$.

Lemma 2.4. Let ${ }_{A} M_{B}$ be a bimodule. Then $\mathscr{S}(A, M, B)$ is a resolving subcategory of $\Lambda$-mod if and only if $M_{B}$ is projective.

Proof. By Lemma 2.1 it suffices to prove that $\mathscr{S}(A, M, B)$ is closed under kernels of epimorphisms if and only if $M_{B}$ is projective. Suppose that $M_{B}$ is projective. Let $f=\left[\begin{array}{c}f_{1} \\ f_{2}\end{array}\right]:\left[\begin{array}{c}X_{1} \\ Y_{1}\end{array}\right]_{\phi_{1}} \rightarrow\left[\begin{array}{c}X_{2} \\ Y_{2}\end{array}\right]_{\phi_{2}}$ be an epimorphism in $\Lambda$-mod with both $\left[\begin{array}{c}X_{1} \\ Y_{1}\end{array}\right]_{\phi_{1}}$ and $\left[\begin{array}{c}X_{2} \\ Y_{2}\end{array}\right]_{\phi_{2}}$ in $\mathscr{S}(A, M, B)$. So $X_{1} \stackrel{f_{1}}{\rightarrow} X_{2}$ and $Y_{1} \stackrel{f_{2}}{\rightarrow} Y_{2}$ are epic with $\operatorname{Ker}\left(f_{1}\right) \stackrel{i}{\hookrightarrow} X_{1}$ and $\operatorname{Ker}\left(f_{2}\right) \stackrel{j}{\hookrightarrow} Y_{1}$. Since $M_{B}$ is projective, we get the commutative diagram with exact rows:

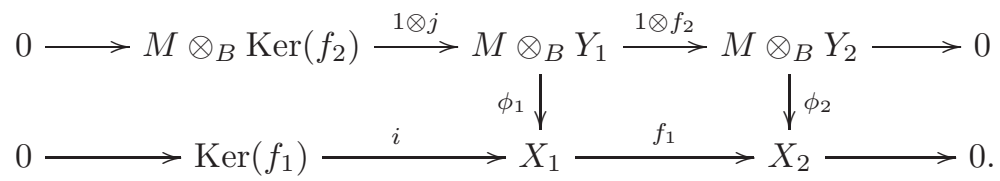

Thus there is a unique $A$-map $\phi: M \otimes_{B} \operatorname{Ker}\left(f_{2}\right) \rightarrow \operatorname{Ker}\left(f_{1}\right)$ such that $i \phi=\phi_{1}(1 \otimes j)$. It is clearly that $\phi$ is monic, and hence $\operatorname{Ker}(f)=\left[\begin{array}{l}\operatorname{Ker}\left(f_{1}\right) \\ \operatorname{Ker}\left(f_{2}\right)\end{array}\right]_{\phi} \in \mathscr{S}(A, M, B)$.

Conversely, suppose that $\mathscr{S}(A, M, B)$ is closed under kernels of epimorphisms. We claim that $M \otimes_{B}-$ is an exact functor. In fact, let $0 \rightarrow K \stackrel{j}{\rightarrow} Y_{1} \stackrel{f}{\rightarrow} Y_{2} \rightarrow 0$ be an arbitrary exact sequence of $B$-modules. Then $\left[\begin{array}{c}1 \otimes f \\ f\end{array}\right]:\left[\begin{array}{c}M \otimes_{B} Y_{1} \\ Y_{1}\end{array}\right]_{\mathrm{Id}} \longrightarrow\left[\begin{array}{c}M \otimes_{B} Y_{2} \\ Y_{2}\end{array}\right]_{\mathrm{Id}}$ is an epimorphism in $\Lambda$-mod with $\left[\begin{array}{c}M \otimes_{B} Y_{i} \\ Y_{i}\end{array}\right]_{\mathrm{Id}} \in \mathscr{S}(A, M, B)(i=1,2)$ and $\operatorname{Ker}\left[\begin{array}{c}1 \otimes f \\ f\end{array}\right]=\left[\begin{array}{c}\operatorname{Ker}(1 \otimes f) \\ K\end{array}\right]_{\phi}$, where $M \otimes_{B} K \stackrel{\phi}{\longrightarrow} \operatorname{Ker}(1 \otimes f)$ is the unique $A$-map such that $\sigma \phi=1 \otimes j$, and $\sigma: \operatorname{Ker}(1 \otimes f) \hookrightarrow M \otimes_{B} Y_{1}$ is the embedding. Since $\mathscr{S}(A, M, B)$ is closed under kernels of epimorphisms, $[\underset{K}{\operatorname{Ker}(1 \otimes f)}]_{\phi} \in \mathscr{S}(A, M, B)$, i.e., $\phi$ is monic, and thus $1 \otimes j$ is monic. This proves the claim and hence $M_{B}$ is flat. Since $B$ is an Artin algebra and $M_{B}$ is finitely generated, it follows that $M_{B}$ is projective.

Lemma 2.5. ([XZ], Lemma 1.2) For $X \in A$-mod and $Y \in B$-mod, we have

(1) $\operatorname{Ext}_{\Lambda}^{1}\left(\left[\begin{array}{l}0 \\ Y\end{array}\right],\left[\begin{array}{c}X \\ 0\end{array}\right]\right) \cong \operatorname{Hom}_{A}\left(M \otimes_{B} Y, X\right)$.

(2) If ${ }_{A} I$ is an injective $A$-module, then $\operatorname{Ext}_{\Lambda}^{i+1}\left(\left[\begin{array}{l}0 \\ Y\end{array}\right],\left[\begin{array}{l}I \\ 0\end{array}\right]\right) \cong \operatorname{Ext}_{B}^{i}\left(Y, \operatorname{Hom}_{A}(M, I)\right)$ for $i \geq 0$.

Proof. For the convenience we include a short justification.

(1) Let $0 \rightarrow K \stackrel{i}{\rightarrow} Q \stackrel{p}{\rightarrow} Y \rightarrow 0$ be an exact sequence with $Q$ a projective left $B$-module. Then $0 \rightarrow\left[\begin{array}{c}M \otimes Q \\ K\end{array}\right]_{1 \otimes i} \stackrel{\left[\begin{array}{l}1 \\ i\end{array}\right]}{\longrightarrow}\left[\begin{array}{c}M \otimes Q \\ Q\end{array}\right]_{\mathrm{Id}} \stackrel{\left[\begin{array}{l}0 \\ p\end{array}\right.}{\longrightarrow}\left[\begin{array}{l}0 \\ Y\end{array}\right] \rightarrow 0$ is an exact sequence with $\left[\begin{array}{c}M \otimes Q \\ Q\end{array}\right]_{\text {Id }}$ a projective left $\Lambda$-module. Applying $\operatorname{Hom}_{\Lambda}\left(-,\left[\begin{array}{c}X \\ 0\end{array}\right]\right)$, since $\operatorname{Hom}_{\Lambda}\left(\left[\begin{array}{c}M \otimes Q \\ Q\end{array}\right]_{\mathrm{Id}},\left[\begin{array}{c}X \\ 0\end{array}\right]\right)=0$, we see

$$
\begin{aligned}
\operatorname{Ext}_{\Lambda}^{1}\left(\left[\begin{array}{l}
0 \\
Y
\end{array}\right],\left[\begin{array}{l}
X \\
0
\end{array}\right]\right) & \cong \operatorname{Hom}_{A}\left(\left[\begin{array}{c}
M \otimes Q \\
K
\end{array}\right]_{1 \otimes i},\left[\begin{array}{l}
X \\
0
\end{array}\right]\right)=\left\{f \in \operatorname{Hom}_{A}\left(M \otimes_{B} Q, X\right) \mid f(1 \otimes i)=0\right\} \\
& \cong \operatorname{Hom}_{A}\left(M \otimes_{B} Y, X\right) .
\end{aligned}
$$

(2) If $i=0$ then the assertion follows from (1) and the Tensor-Hom adjunction isomorphism. Let $i \geq 1$. Using the abbreviation $(M, I)=\operatorname{Hom}_{A}(M, I)$, by the exact sequence $0 \rightarrow\left[\begin{array}{l}I \\ 0\end{array}\right] \rightarrow\left[\begin{array}{c}I \\ (M, I)\end{array}\right]_{\varphi} \rightarrow\left[\begin{array}{c}0 \\ (M, I)\end{array}\right] \rightarrow 0$, 
we get the exact sequence

$$
\operatorname{Ext}_{\Lambda}^{i}\left(\left[\begin{array}{c}
0 \\
Y
\end{array}\right],\left[\left(\begin{array}{c}
I \\
(M, I)
\end{array}\right]_{\varphi}\right) \longrightarrow \operatorname{Ext}_{\Lambda}^{i}\left(\left[\begin{array}{l}
0 \\
Y
\end{array}\right],\left[\begin{array}{c}
0 \\
(M, I)
\end{array}\right]\right) \longrightarrow \operatorname{Ext}_{\Lambda}^{i+1}\left(\left[\begin{array}{c}
0 \\
Y
\end{array}\right],\left[\begin{array}{l}
I \\
0
\end{array}\right]\right) \longrightarrow \operatorname{Ext}_{\Lambda}^{i+1}\left(\left[\begin{array}{c}
0 \\
Y
\end{array}\right],\left[\begin{array}{c}
I \\
(M, I)
\end{array}\right]_{\varphi}\right)\right.
$$

Since $[\stackrel{I}{(M, I)}]_{\varphi}$ is an injective $\Lambda$-module, we have $\operatorname{Ext}_{\Lambda}^{i+1}\left(\left[\begin{array}{c}0 \\ Y\end{array}\right],\left[\begin{array}{l}I \\ 0\end{array}\right]\right) \cong \operatorname{Ext}_{\Lambda}^{i}\left(\left[\begin{array}{c}0 \\ Y\end{array}\right],\left(\begin{array}{c}0 \\ (M, I)\end{array}\right]\right)$, and then the assertion follows from $\operatorname{Ext}_{\Lambda}^{i}\left(\left[\begin{array}{c}0 \\ Y\end{array}\right],\left[\begin{array}{c}0 \\ (M, I)\end{array}\right]\right) \cong \operatorname{Ext}_{B}^{i}\left(Y, \operatorname{Hom}_{A}(M, I)\right)$, by using the injective resolution of $\operatorname{Hom}_{A}(M, I)$.

Lemma 2.6. If $M_{B}$ projective, then $\mathscr{S}(A, M, B)=\perp\left[\begin{array}{c}\mathrm{D}\left(A_{A}\right) \\ 0\end{array}\right]$.

Proof. We need to prove that $\left[\begin{array}{c}X \\ Y\end{array}\right]_{\phi} \in \perp\left[\begin{array}{c}\mathrm{D}\left(A_{A}\right) \\ 0\end{array}\right]$ if and only if $\left[\begin{array}{c}X \\ Y\end{array}\right]_{\phi} \in \mathscr{S}(A, M, B)$. Since $M_{B}$ is projective, $\operatorname{Hom}_{A}\left(M, \mathrm{D}\left(A_{A}\right)\right) \cong \mathrm{D} M$ is an injective left $B$-module, and hence $\operatorname{Ext}_{B}^{i}\left(Y, \operatorname{Hom}_{A}\left(M, \mathrm{D}\left(A_{A}\right)\right)\right)=0$ for all $i \geq 1$. Applying $\operatorname{Hom}_{\Lambda}\left(-,\left[\begin{array}{c}\mathrm{D}\left(A_{A}\right) \\ 0\end{array}\right]\right)$ to the exact sequence $0 \rightarrow\left[\begin{array}{c}X \\ 0\end{array}\right] \rightarrow\left[\begin{array}{c}X \\ Y\end{array}\right]_{\phi} \rightarrow\left[\begin{array}{c}0 \\ Y\end{array}\right] \rightarrow 0$, by Lemma 2.5 we get the commutative diagram with the upper row being exact

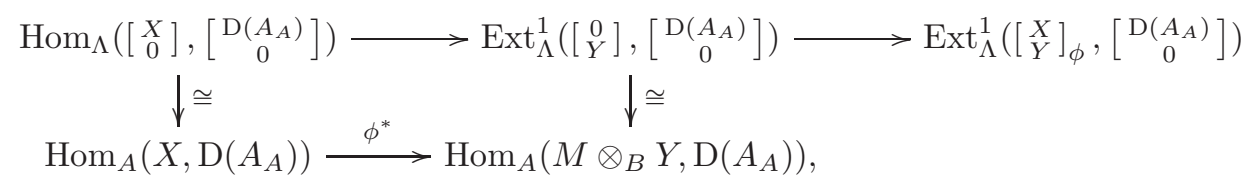

and the following exact sequence for $i \geq 1$

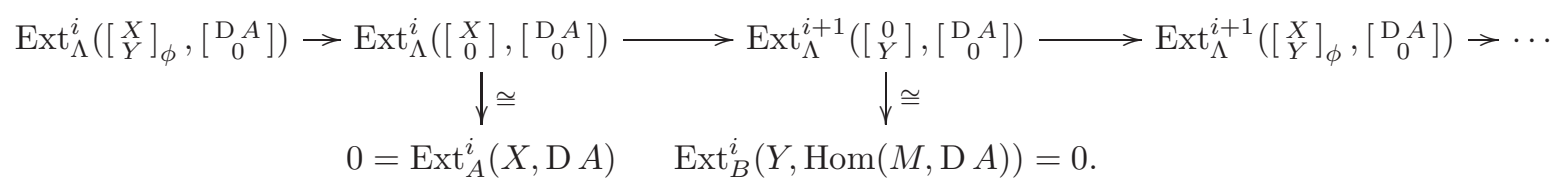

So $\left[\begin{array}{c}X \\ Y\end{array}\right]_{\phi} \in \perp\left[\begin{array}{c}\mathrm{D}\left(A_{A}\right) \\ 0\end{array}\right]$ if and only if $\phi^{*}: \operatorname{Hom}_{A}\left(X, \mathrm{D}\left(A_{A}\right)\right) \rightarrow \operatorname{Hom}_{A}\left(M \otimes_{B} Y, \mathrm{D}\left(A_{A}\right)\right)$ is an epimorphism, and if and only if $\phi: M \otimes_{B} Y \rightarrow X$ is monic.

2.3. Proof of Theorem 1.1. (1) By Lemma 2.4 we have the implications (i) $\Longleftrightarrow$ (ii).

(iii) $\Longrightarrow$ (ii) : Since $\mathscr{S}(A, M, B)={ }^{\perp} T$, it is clear that $\mathscr{S}(A, M, B)$ is a resolving subcategory of $\Lambda$-mod.

(i) $\Longrightarrow$ (iii) : Since $M_{B}$ is projective, $\operatorname{Hom}_{A}\left(M, \mathrm{D}\left(A_{A}\right)\right) \cong \mathrm{D} M$ is an injective left $B$-module, and hence $\left[\operatorname{Hom}_{A}\left(\stackrel{0}{M}, \mathrm{D}\left(A_{A}\right)\right)\right]$ is an injective left $\Lambda$-module. By the exact sequence in $\Lambda$-mod

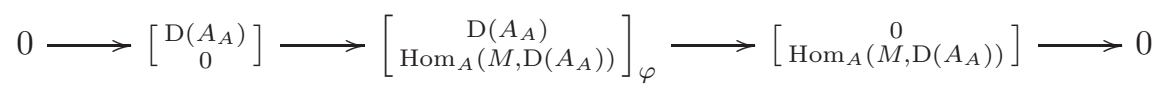

we see that inj. $\operatorname{dim}_{\Lambda}\left[\begin{array}{c}\mathrm{D}\left(A_{A}\right) \\ 0\end{array}\right] \leq 1$.

Let $\alpha: \mathrm{D}\left(B_{B}\right) \rightarrow \operatorname{Hom}_{A}\left(M, E_{\mathrm{D}\left(B_{B}\right)}\right)$ be the image of $e \in \operatorname{Hom}_{A}\left(M \otimes_{B} \mathrm{D}\left(B_{B}\right), E_{\mathrm{D}\left(B_{B}\right)}\right)$ under the adjunction isomorphism

$$
\operatorname{Hom}_{A}\left(M \otimes_{B} \mathrm{D}\left(B_{B}\right), E_{\mathrm{D}\left(B_{B}\right)}\right) \cong \operatorname{Hom}_{B}\left(\mathrm{D}\left(B_{B}\right), \operatorname{Hom}_{A}\left(M, E_{\mathrm{D}\left(B_{B}\right)}\right)\right) .
$$

By the naturalness of the adjunction isomorphisms we have the commutative diagram

$$
\begin{aligned}
& \operatorname{Hom}_{A}\left(M \otimes_{B} \operatorname{Hom}_{A}\left(M, E_{\mathrm{D}\left(B_{B}\right)}\right), E_{\mathrm{D}\left(B_{B}\right)}\right) \stackrel{\cong}{\longrightarrow} \operatorname{Hom}_{B}\left(\operatorname{Hom}_{A}\left(M, E_{\mathrm{D}\left(B_{B}\right)}\right), \operatorname{Hom}_{A}\left(M, E_{\mathrm{D}\left(B_{B}\right)}\right)\right) \\
& \downarrow\left(\operatorname{Id} \otimes_{B} \alpha, E_{\mathrm{D}\left(B_{B}\right)}\right) \quad \downarrow\left(\alpha,\left(M, E_{\mathrm{D}\left(B_{B}\right)}\right)\right) \\
& \operatorname{Hom}_{A}\left(M \otimes_{B} \mathrm{D}\left(B_{B}\right), E_{\mathrm{D}\left(B_{B}\right)}\right) \simeq \operatorname{Hom}_{B}\left(\mathrm{D}\left(B_{B}\right), \operatorname{Hom}_{A}\left(M, E_{\mathrm{D}\left(B_{B}\right)}\right)\right) .
\end{aligned}
$$


Let $\varphi: \operatorname{Hom}_{A}\left(M \otimes_{B} \operatorname{Hom}_{A}\left(M, E_{\mathrm{D}\left(B_{B}\right)}\right), \mathrm{D}\left(B_{B}\right)\right)$ be the involution map. By the above commutative diagram we can get $\varphi\left(1 \otimes_{B} \alpha\right)=e$. So we get a $\Lambda$-map $\left[\begin{array}{l}1 \\ \alpha\end{array}\right]:\left[\begin{array}{c}E_{\mathrm{D}\left(B_{B}\right)} \\ \mathrm{D}\left(B_{B}\right)\end{array}\right]_{e} \longrightarrow\left[\begin{array}{c}E_{\mathrm{D}\left(B_{B}\right)} \\ \operatorname{Hom}_{A}\left(M, E_{\mathrm{D}\left(B_{B}\right)}\right)\end{array}\right]_{\varphi}$, and we have the exact sequence in $\Lambda$-mod

$$
0 \longrightarrow\left[\begin{array}{c}
E_{\mathrm{D}\left(B_{B}\right)} \\
\mathrm{D}\left(B_{B}\right)
\end{array}\right]_{e} \stackrel{\left(\begin{array}{l}
{\left[\begin{array}{l}
1 \\
\alpha \\
{[0} \\
1
\end{array}\right]} \\
\longrightarrow
\end{array}\right)}{\longrightarrow}\left[\begin{array}{c}
E_{\mathrm{D}\left(B_{B}\right)} \\
\operatorname{Hom}_{A}\left(M, E_{\mathrm{D}\left(B_{B}\right)}\right)
\end{array}\right]_{\varphi} \oplus\left[\begin{array}{c}
0 \\
\mathrm{D}\left(B_{B}\right)
\end{array}\right] \stackrel{\left(\left[\begin{array}{l}
0 \\
1
\end{array}\right],\left[\begin{array}{c}
0 \\
-\alpha
\end{array}\right]\right)}{\longrightarrow}\left[\begin{array}{c}
0 \\
\operatorname{Hom}_{A}\left(M, E_{\mathrm{D}\left(B_{B}\right)}\right)
\end{array}\right] \longrightarrow
$$

Since $E_{\mathrm{D}\left(B_{B}\right)}$ is an injective left $A$-module, it follows that $\operatorname{Hom}_{A}\left(M, E_{\mathrm{D}\left(B_{B}\right)}\right) \in \operatorname{add}\left(\operatorname{Hom}_{A}\left(M, \mathrm{D}\left(A_{A}\right)\right)\right)$, so $\operatorname{Hom}_{A}\left(M, E_{\mathrm{D}\left(B_{B}\right)}\right)$ is an injective left $B$-module, and hence $\left[\operatorname{Hom}_{A}\left(\stackrel{0}{\left(M, E_{\mathrm{D}\left(B_{B}\right)}\right)}\right]\right.$ is an injective $\Lambda$-module. This shows inj. $\operatorname{dim}_{\Lambda}\left[\begin{array}{c}E_{\mathrm{D}\left(B_{B}\right)} \\ \mathrm{D}\left(B_{B}\right)\end{array}\right]_{e} \leq 1$. Thus inj. $\operatorname{dim}_{\Lambda} T=\operatorname{inj} \cdot \operatorname{dim}_{\Lambda}\left(\left[\begin{array}{c}\mathrm{D}\left(A_{A}\right) \\ 0\end{array}\right] \oplus\left[\begin{array}{c}E_{\mathrm{D}\left(B_{B}\right)} \\ \mathrm{D}\left(B_{B}\right)\end{array}\right]_{e}\right) \leq 1$.

By Proposition 2.2(2), $T$ is an injective object of $\mathscr{S}(A, M, B)$, so $\operatorname{Ext}_{\Lambda}^{1}(T, T)=0$. It is clear that the number of the pairwise non-isomorphic indecomposable direct summands of $T$ is the number of the simple $\Lambda$-modules. So ${ }_{\Lambda} T$ is a cotilting $\Lambda$-module.

Since $T$ is an injective object of $\mathscr{S}(A, M, B)$ and inj. $\operatorname{dim}_{\Lambda} T \leq 1$, we have $\mathscr{S}(A, M, B) \subseteq{ }^{\perp} T$. By Lemma 2.6. $\mathscr{S}(A, M, B)=\perp\left[\begin{array}{c}D\left(A_{A}\right) \\ 0\end{array}\right] \supseteq{ }^{\perp} T$. Thus $\mathscr{S}(A, M, B)={ }^{\perp} T$.

If there is another cotilting $\Lambda$-module $L$ such that $\mathscr{S}(A, M, B)={ }^{\perp} L$. Then $T \oplus L$ is also a cotilting $\Lambda$-module. By comparing the number of pairwise non-isomorphic indecomposable direct summands of $T \oplus L$ and $T$, we see the uniqueness of $T$, up to the multiplicities of indecomposable direc summands.

(2) The following construction is from C. Ringel and M. Schmidmeier [RS2]. Let $\left[\begin{array}{l}X \\ Y\end{array}\right]_{\phi} \in \Lambda$-mod. Define $\operatorname{Mimo}(\phi)$ to be the $\Lambda$-module $[\underset{Y}{X \oplus \operatorname{IKer}(\phi)}]_{\left[\begin{array}{l}\phi \\ e\end{array}\right]}$, where $e: M \otimes_{B} Y \longrightarrow \operatorname{IKer}(\phi)$ is an extension of the injective envelope $\operatorname{Ker}(\phi) \hookrightarrow \operatorname{IKer}(\phi)$. Then it is clear that $\operatorname{Mimo}(\phi)$ is well-defined (i.e., independent of the choice of $e$ ) and it is in $\mathscr{S}(A, M, B)$. For any $\left[\begin{array}{l}X \\ Y\end{array}\right]_{\phi} \in \Lambda$-mod, by the similar argument as in [RS2, Prop. 2.4], one can see that $\left[\begin{array}{c}(1,0) \\ 1\end{array}\right]: \operatorname{Mimo}(\phi)=[\underset{Y}{X \oplus \operatorname{Ker}(\phi)}]_{\left[\begin{array}{l}\phi \\ e\end{array}\right]} \longrightarrow\left[\begin{array}{c}X \\ Y\end{array}\right]_{\phi}$ is a minimal right $\mathscr{S}(A, M, B)$-approximation of $\left[\begin{array}{l}X \\ Y\end{array}\right]_{\phi}$. Thus $\mathscr{S}(A, M, B)$ is a contravariantly finite subcategory of $\Lambda$-mod.

By [KS, Corol. 0.3], a resolving contravariantly finite subcategory of $\Lambda$-mod is functorially finite, and by [AS, Thm. 2.4], an extension-closed functorially finite subcategory of $\Lambda$-mod has Auslander-Reiten sequences. Thus, if $M_{B}$ is projective, then by $(1), \mathscr{S}(A, M, B)$ is functorially finite in $\Lambda$-mod, and hence $\mathscr{S}(A, M, B)$ has Auslander-Reiten sequences.

2.4. Recall that each right $\Lambda$-module is identified with a triple $(X, Y)_{\phi}$, where $X \in \bmod A, Y \in \bmod B$, and $\phi: X \otimes_{A} M \rightarrow Y$ is a right $B$-map; and a right $\Lambda$-map is identified with a pair $\left(f_{1}, f_{2}\right):\left(X_{1}, Y_{1}\right)_{\phi_{1}} \rightarrow$ $\left(X_{2}, Y_{2}\right)_{\phi_{2}}$, where $f_{1}: X_{1} \rightarrow X_{2}$ is an $A$-map and $f_{2}: Y_{1} \rightarrow Y_{2}$ a $B$-map, such that $f_{2} \phi_{1}=\phi_{2}\left(f_{1} \otimes 1\right)$. The injective right $\Lambda$-modules are exactly $(I, 0)$ and $\left(\operatorname{Hom}_{B}(M, J), J\right)_{\varphi}$, where $I$ (resp. $\left.J\right)$ runs over the injective right $A$-modules (resp. $B$-modules), and $\varphi: \operatorname{Hom}_{B}(M, J) \otimes_{A} M \rightarrow J$ is the involution map given by $\varphi(f \otimes m)=f(m)$. See [ARS, p.73].

All the results obtained so far have the right module versions. We only write down what is needed later. The right module version of $\mathscr{S}(A, M, B)$ is $\mathscr{S}(A, M, B)_{r}$, which is the subcategory of $\bmod \Lambda$ consisting of the triple $(U, V)_{\phi}$, where $X \in \bmod A, Y \in \bmod B$, and $\phi: X \otimes_{A} M \rightarrow Y$ is a monic right $B$-map. Then $\mathscr{S}(A, M, B)_{r}$ is a resolving subcategory of $\Lambda$-mod if and only if ${ }_{A} M$ projective, and if and only if 
$\mathscr{S}(A, M, B)_{r}={ }^{\perp}\left(\mathrm{D}\left({ }_{A} A\right), 0\right)$. The following result is only a part of the right module version of Theorem 1.1 which is what we will need later.

A right module version of Theorem 1.1. Let ${ }_{A} M_{B}$ be a bimodule with ${ }_{A} M$ projective. Then $U_{\Lambda}:=$ $\left(\mathrm{D}\left({ }_{A} A\right), E_{\mathrm{D}\left({ }_{A} A\right)}\right)_{e} \oplus\left(0, \mathrm{D}\left({ }_{B} B\right)\right)$ is a unique cotilting right $\Lambda$-module, up to multiplicities of indecomposable direct summands, such that $\mathscr{S}(A, M, B)_{r}={ }^{\perp}\left(U_{\Lambda}\right)$, where $E_{\mathrm{D}\left({ }_{A} A\right)}$ is an injective envelope of $\mathrm{D}\left({ }_{A} A\right) \otimes_{A} M$ with embedding e $: \mathrm{D}\left({ }_{A} A\right) \otimes_{A} M \hookrightarrow E_{\mathrm{D}\left({ }_{A} A\right)}$.

In particular, if $\mathrm{D}\left({ }_{A} A\right) \otimes_{A} M$ is an injective right $B$-module, then $U_{\Lambda}=\left(\mathrm{D}\left({ }_{A} A\right), \mathrm{D}\left({ }_{A} A\right) \otimes_{A} M\right)_{\operatorname{Id}} \oplus$ $\left(0, \mathrm{D}\left({ }_{B} B\right)\right)$ is a unique cotilting right $\Lambda$-module, up to multiplicities of indecomposable direct summands, such that $\mathscr{S}(A, M, B)_{r}={ }^{\perp}\left(U_{\Lambda}\right)$.

\section{Proof of Theorem 1.3}

3.1. Let $\mathscr{A}, \mathscr{B}, \mathscr{C}$ be additive categories. The diagram of functors

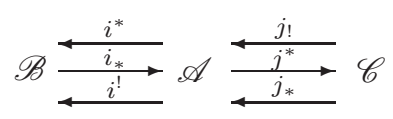

is a recollement of $\mathscr{A}$ relative to $\mathscr{B}$ and $\mathscr{C}$, if the conditions (R1),(R2),(R3) are satisfied:

(R1) $\left(i^{*}, i_{*}\right),\left(i_{*}, i^{!}\right),\left(j_{1}, j^{*}\right)$ and $\left(j^{*}, j_{*}\right)$ are adjoint pairs;

(R2) $i_{*}, j_{\text {! }}$ and $j_{*}$ are fully faithful;

(R3) $\operatorname{Im} i_{*}=\operatorname{Ker} j^{*}$.

Since the functors in an adjoint pair between additive categories are additive functors, all the six functors in a recollement of additive categories are additive.

3.2. The following fact is easy.

Lemma 3.1. Let $\mathscr{A}$ and $\mathscr{B}$ be additive categories with subcategories $\mathscr{X}$ and $\mathscr{Y}$, respectively, $(F, G)$ an adjoint pair with $F: \mathscr{A} \rightarrow \mathscr{B}$ and $G: \mathscr{B} \rightarrow \mathscr{A}$. If $F \mathscr{X} \subseteq \mathscr{Y}$ and $G \mathscr{Y} \subseteq \mathscr{X}$, then there is an induced adjoint pair $(\bar{F}, \bar{G})$ with $\bar{F}: \mathscr{A} / \mathscr{X} \rightarrow \mathscr{B} / \mathscr{Y}$ and $\bar{G}: \mathscr{B} / \mathscr{Y} \rightarrow \mathscr{A} / \mathscr{X}$.

Let $\Lambda=\left[\begin{array}{cc}A & M \\ 0 & B\end{array}\right]$ be an Artin algebra. Define five functors as follows.

$$
\begin{array}{ll}
i^{*}: \mathscr{S}(A, M, B) \rightarrow A \text {-mod, }\left[\begin{array}{c}
X \\
Y
\end{array}\right]_{\phi} \mapsto \operatorname{Coker}(\phi) ; & j !: B \text {-mod } \rightarrow \mathscr{S}(A, M, B), Y \mapsto\left[\begin{array}{c}
M \otimes_{B} Y \\
Y
\end{array}\right]_{\mathrm{Id}} ; \\
i_{*}: A-\bmod \rightarrow \mathscr{S}(A, M, B), X \mapsto\left[\begin{array}{c}
X \\
0
\end{array}\right] ; & j^{*}: \mathscr{S}(A, M, B) \rightarrow B \text {-mod, }\left[\begin{array}{l}
X \\
Y
\end{array}\right]_{\phi} \mapsto Y ; \\
i_{!}: \mathscr{S}(A, M, B) \rightarrow A \text {-mod, }\left[\begin{array}{l}
X \\
Y
\end{array}\right]_{\phi} \mapsto X . &
\end{array}
$$

Lemma 3.2. Let $\Lambda=\left[\begin{array}{cc}A & M \\ 0 & B\end{array}\right]$ be an Artin algebra. Then

(1) $\left(i^{*}, i_{*}\right),\left(i_{*}, i^{!}\right)$, and $\left(j_{!}, j^{*}\right)$ are adjoint pairs;

(2) $i_{*}$ and $j_{\text {! }}$ are fully faithful;

(3) $\operatorname{Im} i_{*}=\operatorname{Ker} j^{*}$. 
Proof. (1) For any $[\underset{Y}{X}]_{\phi} \in \mathscr{S}(A, M, B), W \in A$-mod and $V \in B$-mod, we have the isomorphisms:

$$
\begin{aligned}
& \operatorname{Hom}_{A}\left(i^{*}\left(\left[\begin{array}{c}
X \\
Y
\end{array}\right]_{\phi}\right), W\right)=\operatorname{Hom}_{A}(\operatorname{Coker}(\phi), W) \cong \operatorname{Hom}_{\Lambda}\left(\left[\begin{array}{c}
X \\
Y
\end{array}\right]_{\phi},\left[\begin{array}{c}
W \\
0
\end{array}\right]\right)=\operatorname{Hom}_{\Lambda}\left(\left[\begin{array}{c}
X \\
Y
\end{array}\right]_{\phi}, i_{*}(W)\right) ; \\
& \operatorname{Hom}_{\Lambda}\left(i_{*}(W),\left[\begin{array}{c}
X \\
Y
\end{array}\right]_{\phi}\right)=\operatorname{Hom}_{\Lambda}\left(\left[\begin{array}{c}
W \\
0
\end{array}\right],\left[\begin{array}{c}
X \\
Y
\end{array}\right]_{\phi}\right) \cong \operatorname{Hom}_{A}(W, X)=\operatorname{Hom}_{A}\left(W, i_{!}\left(\left[\begin{array}{c}
X \\
Y
\end{array}\right]_{\phi}\right)\right) ; \\
& \operatorname{Hom}_{\Lambda}\left(j_{!}(V),\left[\begin{array}{c}
X \\
Y
\end{array}\right]_{\phi}\right)=\operatorname{Hom}_{\Lambda}\left(\left[\begin{array}{c}
M \otimes_{B} V \\
V
\end{array}\right]_{1},\left[\begin{array}{l}
X \\
Y
\end{array}\right]_{\phi}\right) \cong \operatorname{Hom}_{B}(V, Y)=\operatorname{Hom}_{B}\left(V, j^{*}\left(\left[\begin{array}{l}
X \\
Y
\end{array}\right]_{\phi}\right)\right) .
\end{aligned}
$$

These show that $\left(i^{*}, i_{*}\right),\left(i_{*}, i^{!}\right)$, and $\left(j_{!}, j^{*}\right)$ are adjoint pairs.

(2) For any $X_{1}, X_{2} \in A$-mod and $Y_{1}, Y_{2} \in B$-mod, we have the isomorphisms:

$$
\begin{aligned}
& \operatorname{Hom}_{\Lambda}\left(i_{*}\left(X_{1}\right), i_{*}\left(X_{2}\right)\right)=\operatorname{Hom}_{\Lambda}\left(\left[\begin{array}{c}
X_{1} \\
0
\end{array}\right],\left[\begin{array}{c}
X_{2} \\
0
\end{array}\right]\right) \cong \operatorname{Hom}_{A}\left(X_{1}, X_{2}\right) \\
& \operatorname{Hom}_{\Lambda}\left(j_{!}\left(Y_{1}\right), j_{!}\left(Y_{2}\right)\right)=\operatorname{Hom}_{\Lambda}\left(\left[\begin{array}{c}
M \otimes_{B} Y_{1} \\
Y_{1}
\end{array}\right]_{\mathrm{Id}},\left[\begin{array}{c}
M \otimes_{B} Y_{2} \\
Y_{2}
\end{array}\right]_{\mathrm{Id}}\right) \cong \operatorname{Hom}_{B}\left(Y_{1}, Y_{2}\right) .
\end{aligned}
$$

These show that $i_{*}$ and $j$ ! are fully faithful.

(3) This is clear.

Suppose that ${ }_{A} M_{B}$ satisfies the condition (IP). By Proposition 2.2 the injective objects of $\mathscr{S}(A, M, B)$ are exactly $\left[\begin{array}{c}I \\ 0\end{array}\right]$ and $\left[\underset{J}{M \otimes_{B} J}\right]_{1}$, where $I$ runs over injective $A$-modules, and $J$ runs over injective $B$-modules. Thus, by the constructions all the functors $i^{*}, i_{*}, i_{!}, j_{!}$and $j^{*}$ preserve injective objects. It follows from Lemmas 3.1 and 3.2 that there are the induced functors:

$$
\begin{aligned}
& \overline{i^{*}}: \overline{\mathscr{S}(A, M, B)} \rightarrow A-\overline{\mathrm{mod}}, \quad\left[\begin{array}{c}
X \\
Y
\end{array}\right]_{\phi} \mapsto \operatorname{Coker}(\phi) ; \quad \overline{j !}: B-\overline{\bmod } \rightarrow \overline{\mathscr{S}(A, M, B)}, \quad Y \mapsto\left[{ }_{Y}^{M \otimes_{B} Y}\right]_{\mathrm{Id}} ; \\
& \overline{i_{*}}: A \text { - } \overline{\bmod } \rightarrow \overline{\mathscr{S}(A, M, B)}, \quad X \mapsto\left[\begin{array}{c}
X \\
0
\end{array}\right] ; \quad \overline{j^{*}}: \overline{\mathscr{S}(A, M, B)} \rightarrow B-\overline{\bmod }, \quad\left[\begin{array}{c}
X \\
Y
\end{array}\right]_{\phi} \mapsto Y ; \\
& \overline{i_{!}}: \overline{\mathscr{S}(A, M, B)} \rightarrow A-\overline{\mathrm{mod}}, \quad\left[\begin{array}{l}
X \\
Y
\end{array}\right]_{\phi} \mapsto X
\end{aligned}
$$

such that the following fact holds:

Lemma 3.3. If ${ }_{A} M_{B}$ satisfies the condition (IP), then $\left(\overline{i^{*}}, \overline{i_{*}}\right),\left(\overline{i_{*}}, \overline{i^{!}}\right)$and $\left(\overline{j_{1}}, \overline{j^{*}}\right)$ are adjoint pairs.

Moreover, if in addition both $A$ and $B$ are selfinjective algebras, then all the functors $\overline{i^{*}}, \overline{i_{*}}, \overline{i^{!}}, \overline{j_{!}}, \overline{j^{*}}$ are triangle functors between triangulated categories.

Proof. We only need to justify the last assertion. In this case, both $A$-mod and $B$-mod are triangulated categories. By Example 1.5(7), ${ }_{A} M$ is projective; and then by Corollary 2.3. $\mathscr{S}(A, M, B)$ is a Frobenius category, hence $\overline{\mathscr{S}(A, M, B)}$ is also a triangulated category. See [H1, p.16]. Recall the distinguished triangles in the stable category of a Frobenius category. Each exact sequence $0 \rightarrow X_{1} \stackrel{u}{\rightarrow} X_{2} \stackrel{v}{\rightarrow} X_{3} \rightarrow 0$ in $A$-mod gives rise to a distinguished triangle $X_{1} \stackrel{u}{\rightarrow} X_{2} \stackrel{v}{\rightarrow} X_{3} \rightarrow X$ [1] of $A$-mod, and conversely, each distinguished triangle of $A-\overline{\mathrm{mod}}$ is of this form up to an isomorphism of triangles (see [H1], Chap. 1, Sect. 2; also [CZ], Lemma 1.2). Since the functor $i_{*}: A$-mod $\rightarrow \mathscr{S}(A, M, B)$ given by $X \mapsto\left[\begin{array}{l}X \\ 0\end{array}\right]$ preserves exact sequences, $\overline{i_{*}}: A$ - $\overline{\bmod } \rightarrow \overline{\mathscr{S}(A, M, B)}$ preserves the distinguished triangles, i.e., $\overline{i_{*}}$ is a triangle functor. Note that in an adjoint pair $(F, G)$ between triangulated categories, $F$ is a triangle functor if and only if so is $G$ ([Ke, 6.7], [N, p.179]). Thus, $\overline{i^{*}}$ and $\overline{i_{1}}$ are triangle functors.

Similarly, $\overline{j^{*}}: \overline{\mathscr{S}(A, M, B)} \rightarrow B$ - $\overline{\bmod }$ is a triangle functor, and then so is $\overline{j_{!}}$.

The following lemma is crucial in the proof of Theorem 1.3

Lemma 3.4. Assume that ${ }_{A} M_{B}$ satisfies the condition (IP). Then there exists a fully faithful functor $\overline{j_{*}}: B-\overline{\bmod } \rightarrow \overline{\mathscr{S}(A, M, B)}$ such that $\left(\overline{j^{*}}, \overline{j_{*}}\right)$ is an adjoint pair. 
Proof. The following construction is similar as [Z2, Thm. 3.5]. Define a functor $j_{*}: B$-mod $\rightarrow \overline{\mathscr{S}(A, M, B)}$ as follows. For $Y \in B$-mod, define $j_{*}(Y):=\left[\begin{array}{c}E_{Y} \\ Y\end{array}\right]_{\psi}$, where $E_{Y}$ is an injective envelope of the $A$-module $M \otimes_{B} Y$ with embedding $\psi: M \otimes_{B} Y \rightarrow E_{Y}$. (This is clearly well-defined. One can see this also from the argument below, by taking $h=\mathrm{Id}_{Y}$.) For $h: Y \rightarrow Y^{\prime}$, there is a commutative diagram with exact rows

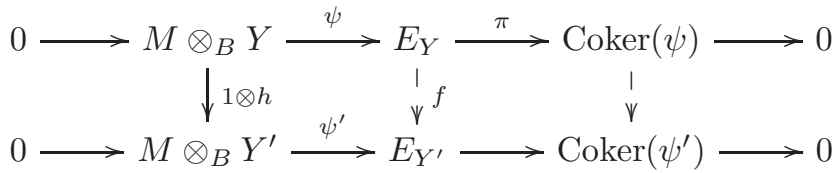

and we define $j_{*}(h):=\overline{\left[\begin{array}{c}f \\ h\end{array}\right]}:\left[\begin{array}{c}E_{Y} \\ Y\end{array}\right]_{\psi} \rightarrow\left[\begin{array}{c}E_{Y^{\prime}} \\ Y^{\prime}\end{array}\right]_{\psi^{\prime}}$. We claim that $j_{*}(h)$ is well-defined, and hence the functor $j_{*}: B-\bmod \rightarrow \overline{\mathscr{S}(A, M, B)}$ is well-defined.

In fact, if there is another $A$-map $f^{\prime}: E_{Y} \rightarrow E_{Y^{\prime}}$ such that $f^{\prime} \psi=\psi^{\prime}(1 \otimes h)$, then $f^{\prime} \psi=f \psi$, i.e., $\left(f-f^{\prime}\right) \psi=0$ and hence $f-f^{\prime}: E_{Y} \rightarrow E_{Y^{\prime}}$ factors through Coker $(\psi)$. Furthermore, $f-f^{\prime}$ factors through an injective envelope $I$ of $\operatorname{Coker}(\psi)$. Then it is clear that $\left[\begin{array}{l}f \\ h\end{array}\right]-\left[\begin{array}{c}f^{\prime} \\ h\end{array}\right]=\left[\begin{array}{c}f-f^{\prime} \\ 0\end{array}\right]:\left[\begin{array}{c}E_{Y} \\ Y\end{array}\right]_{\psi} \rightarrow\left[\begin{array}{c}E_{Y^{\prime}} \\ Y^{\prime}\end{array}\right]_{\psi^{\prime}}$ factors through the injective object $\left[\begin{array}{l}I \\ 0\end{array}\right]$ of $\mathscr{S}(A, M, B)$. So $\overline{\left[\begin{array}{l}f \\ h\end{array}\right]}=\overline{\left[\begin{array}{l}f^{\prime} \\ h\end{array}\right]}$ in $\overline{\mathscr{S}(A, M, B)}$.

Next, we claim that the functor $j_{*}: B$-mod $\rightarrow \overline{\mathscr{S}(A, M, B)}$ induces a functor $\overline{j_{*}}: B$-mod $\rightarrow \overline{\mathscr{S}(A, M, B)}$. For this, assume that $h: Y \rightarrow Y^{\prime}$ factors through an injective $B$-module $J$ via $h=h_{2} h_{1}$ with some $h_{1} \in \operatorname{Hom}_{B}(Y, J)$ and some $h_{2} \in \operatorname{Hom}_{B}\left(J, Y^{\prime}\right)$. Taking an injective envelope $E_{J}$ of the $A$-module $M \otimes_{B} J$ with embedding $\eta: M \otimes_{B} J \rightarrow E_{J}$, then there are $\sigma \in \operatorname{Hom}_{A}\left(E_{Y}, E_{J}\right)$ and $\delta \in \operatorname{Hom}_{A}\left(E_{J}, E_{Y^{\prime}}\right)$, such that $\eta\left(1 \otimes h_{1}\right)=\sigma \psi$ and $\psi^{\prime}\left(1 \otimes h_{2}\right)=\delta \eta$. Thus $f \psi=\psi^{\prime}(1 \otimes h)=\psi^{\prime}\left(1 \otimes h_{2}\right)\left(1 \otimes h_{1}\right)=\delta \eta\left(1 \otimes h_{1}\right)=\delta \sigma \psi$, i.e., $(f-\delta \sigma) \psi=0$. Hence $f-\delta \sigma$ factors through $\operatorname{Coker}(\psi)$ via $f-\delta \sigma=\gamma \pi$ with some $\gamma \in \operatorname{Hom}_{A}\left(\operatorname{Coker}(\psi), E_{Y^{\prime}}\right)$. Consider an injective envelope $I$ of $\operatorname{Coker}(\psi)$ with embedding $\alpha: \operatorname{Coker}(\psi) \rightarrow I$. Then there is a $\beta \in$ $\operatorname{Hom}_{A}\left(I, E_{Y^{\prime}}\right)$ such that $\gamma=\beta \alpha$, and then we have $f=\delta \sigma+\beta \alpha \pi$. We present this process as the diagram:

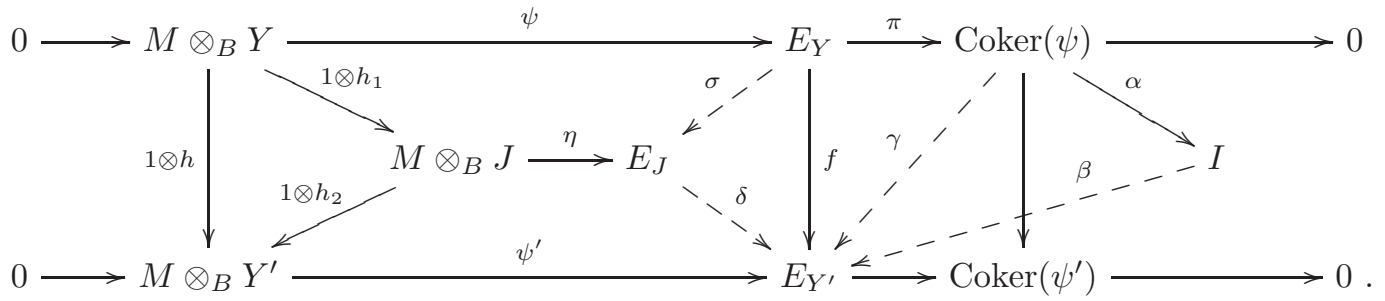

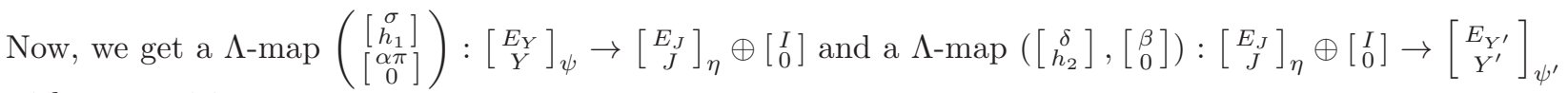
with composition

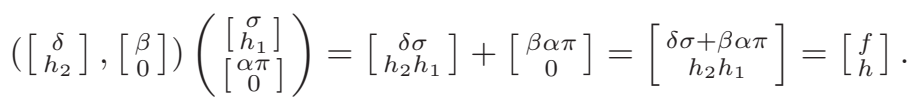

This shows that $\left[\begin{array}{c}f \\ h\end{array}\right]:\left[\begin{array}{c}E_{Y} \\ Y\end{array}\right]_{\psi} \rightarrow\left[\begin{array}{c}E_{Y^{\prime}} \\ Y^{\prime}\end{array}\right]_{\psi^{\prime}}$ factors through the injective object $\left[\begin{array}{c}E_{J} \\ J\end{array}\right]_{\eta} \oplus\left[\begin{array}{l}I \\ 0\end{array}\right]$ of $\mathscr{S}(A, M, B)$, and hence $j_{*}(h)=\overline{\left[\begin{array}{l}f \\ h\end{array}\right]}=0$ in $\overline{\mathscr{S}(A, M, B)}$. Thus $j_{*}$ induces a functor

$$
\overline{j_{*}}: B \overline{\bmod } \rightarrow \overline{\mathscr{S}(A, M, B)}
$$

given by $\overline{j_{*}}(Y):=\left[\begin{array}{c}E_{Y} \\ Y\end{array}\right]_{\psi}$ and $\overline{j_{*}}(\bar{h}):=\overline{\left[\begin{array}{c}f \\ h\end{array}\right]}$. 
By construction it is clear that $\overline{j_{*}}$ is full. For $h: Y \rightarrow Y^{\prime}$, assume that $\overline{j_{*}}(\bar{h})=\overline{\left[\begin{array}{l}f \\ h\end{array}\right]}=0$. Then $\left[\begin{array}{c}f \\ h\end{array}\right]:\left[\begin{array}{c}E_{Y} \\ Y\end{array}\right]_{\psi} \rightarrow\left[\begin{array}{c}E_{Y^{\prime}} \\ Y^{\prime}\end{array}\right]_{\psi^{\prime}}$ factors through an injective object $\left[\begin{array}{c}E_{J} \\ J\end{array}\right]_{\eta} \oplus\left[\begin{array}{c}I \\ 0\end{array}\right]$ of $\mathscr{S}(A, M, B)$, and hence $h: Y \rightarrow Y^{\prime}$ factors through the injective $B$-module $J$. So $\overline{j_{*}}$ is also faithful.

It remains to prove that $\left(\overline{j^{*}}, \overline{j_{*}}\right)$ is an adjoint pair. Thus, for $\left[\begin{array}{r}X \\ Y\end{array}\right]_{\phi} \in \mathscr{S}(A, M, B)$ and $Y^{\prime} \in B$-mod, we need to show that there is a bi-functorial isomorphism $\left.\overline{\operatorname{Hom}}_{B}\left({\overline{j^{*}}}_{\left(\left[\begin{array}{l}X \\ Y\end{array}\right]_{\phi}\right.}\right), Y^{\prime}\right) \cong \overline{\operatorname{Hom}}_{\Lambda}\left(\left[\begin{array}{l}X \\ Y\end{array}\right]_{\phi}, \overline{j_{*}}\left(Y^{\prime}\right)\right)$, i.e.

$$
\overline{\operatorname{Hom}}_{B}\left(Y, Y^{\prime}\right) \cong \overline{\operatorname{Hom}}_{\Lambda}\left(\left[\begin{array}{c}
X \\
Y
\end{array}\right]_{\phi},\left[\begin{array}{c}
E_{Y^{\prime}} \\
Y^{\prime}
\end{array}\right]_{\psi^{\prime}}\right)
$$

where $\psi^{\prime}: M \otimes_{B} Y^{\prime} \rightarrow E_{Y^{\prime}}$ is an injective envelope of $M \otimes_{B} Y^{\prime}$. We claim that the map $\bar{h} \mapsto \overline{\left[\begin{array}{l}f \\ h\end{array}\right]}$ gives such an isomorphism, where $f: X \rightarrow E_{Y^{\prime}}$ is an $A$-map such that $f \phi=\psi^{\prime}(1 \otimes h)$. In fact, a $\Lambda$ map $\left[\begin{array}{l}f \\ h\end{array}\right]:\left[\begin{array}{c}X \\ Y\end{array}\right]_{\phi} \rightarrow\left[\begin{array}{c}E_{Y^{\prime}} \\ Y^{\prime}\end{array}\right]_{\psi^{\prime}}$ factors through an injective object $\left[\begin{array}{c}I \\ 0\end{array}\right] \oplus\left[\begin{array}{c}E_{J} \\ J\end{array}\right]_{\psi}$ of $\mathscr{S}(A, M, B)$ if and only if $h: Y \rightarrow Y^{\prime}$ factors through the injective $B$-module $J$. This shows that the given map above is well-defined and injective; and by the construction it is clearly surjective. This completes the proof.

3.3. Proof of Theorem 1.3. To see the diagram (1.1) forms a recollement of additive categories, by Lemmas 3.3 and 3.4 it remains to prove that $\overline{i_{*}}$ and $\overline{j_{!}}$are fully faithful, and that $\operatorname{Im} \overline{i_{*}}=\operatorname{Ker} \overline{j^{*}}$.

Since ${ }_{A} M_{B}$ satisfies the condition (IP), by Proposition $\left[2.2(2),\left[\begin{array}{l}I \\ 0\end{array}\right]\right.$ and $\left[\begin{array}{c}M \otimes_{J} J \\ J\end{array}\right]_{\mathrm{Id}}$ are injective objects of $\mathscr{S}(A, M, B)$, where $I$ is an injective $A$-module and $J$ is an injective $B$-module. Recall that $\overline{i_{*}}: A$-mod $\rightarrow$ $\overline{\mathscr{S}(A, M, B)}$ is given by $X \mapsto\left[\begin{array}{l}X \\ 0\end{array}\right]$ and $\bar{f} \mapsto \overline{\left[\begin{array}{l}f \\ 0\end{array}\right]}$, and $\overline{j_{!}}: B$ - $\overline{\bmod } \rightarrow \overline{\mathscr{S}(A, M, B)}$ is given by $Y \mapsto\left[{ }_{Y}^{M \otimes_{B} Y}\right]_{\mathrm{Id}}$ and $\bar{h} \mapsto \overline{\left[\begin{array}{c}1 \otimes h \\ h\end{array}\right]}$. It is easy to see that $\overline{i_{*}}$ and $\overline{j_{!}}$are fully faithful.

It is clear that $\operatorname{Ker}\left(\overline{j^{*}}\right)=\left\{\left[\begin{array}{c}X \\ Y\end{array}\right]_{\phi} \in \mathscr{S}(A, M, B) \mid{ }_{B} Y\right.$ injective $\}$. For any $\left[\begin{array}{l}X \\ Y\end{array}\right]_{\phi} \in \operatorname{Ker}\left(\overline{j^{*}}\right)$, by the condition (IP), $M \otimes_{B} Y$ is an injective $A$-module, and hence the exact sequence $0 \rightarrow M \otimes_{B} Y \stackrel{\phi}{\rightarrow} X \rightarrow \operatorname{Coker}(\phi) \rightarrow 0$ splits. So $\left[\begin{array}{r}X \\ Y\end{array}\right]_{\phi} \cong\left[\begin{array}{c}M \otimes_{B} Y \\ Y\end{array}\right]_{\mathrm{Id}} \oplus[\underset{0}{\operatorname{Coker}(\phi)}]$. Since $\left[\begin{array}{c}M \otimes_{B} Y \\ Y\end{array}\right]_{\mathrm{Id}}$ is an injective object of $\mathscr{S}(A, M, B),\left[\begin{array}{l}X \\ Y\end{array}\right]_{\phi}=$ $\left[\begin{array}{c}\operatorname{Coker}(\phi) \\ 0\end{array}\right]$ in $\overline{\mathscr{S}(A, M, B)}$. Thus $\operatorname{Ker} \overline{j^{*}} \subseteq \operatorname{Im} \overline{i_{*}}$, and $\operatorname{Im} \overline{i_{*}}=\operatorname{Ker} \overline{j^{*}}$.

If both $A$ and $B$ are selfinjective, then by Lemma 3.3 , all the functors $\overline{i^{*}}, \overline{i_{*}}, \overline{i^{!}}, \overline{j_{l}}$ and $\overline{j^{*}}$ are triangle functors, and hence $\overline{j_{*}}$ is also a triangle functor. So (1.1) is a recollement of triangulated categories. In this case $\Lambda$ is a Gorenstein algebra and $\mathscr{S}(A, M, B)$ is exactly the category of Gorenstein-projective $\Lambda$-modules ([Z2, Lemma 2.1, Thm. 2.2]), thus $\overline{\mathscr{S}(A, M, B)}$ is exactly the singularity category $\mathcal{D}_{s g}^{b}(\Lambda):=\mathcal{D}^{b}(\Lambda$-mod $) / K^{b}(\operatorname{proj}(\Lambda))($ see R. Buchweitz [Buch, Thm. 4.4.1]; see also [O]). Similarly, $A-\overline{\bmod } \cong \mathcal{D}_{s g}^{b}(A)$ and $B-\overline{\bmod } \cong \mathcal{D}_{s g}^{b}(B)$.

\section{The dual version: the epimorphism category induced by a bimodule}

4.1. We briefly state the dual version for the next section. The epimorphism category $\mathscr{F}(A, M, B)$ induced by a bimodule ${ }_{A} M_{B}$ is the subcategory of $\Lambda$-mod consisting of $\left[\begin{array}{l}X \\ Y\end{array}\right]_{\phi}$ such that $\eta_{Y, X}(\phi): Y \rightarrow \operatorname{Hom}_{A}(M, X)$ is an epic left $B$-map, where

$$
\eta_{Y, X}: \operatorname{Hom}_{A}\left(M \otimes_{B} Y, X\right) \cong \operatorname{Hom}_{B}\left(Y, \operatorname{Hom}_{A}(M, X)\right)
$$

is the adjunction isomorphism. Then $\mathscr{F}(A, M, B)$ contains all the injective $\Lambda$-modules and is closed under direct sums, direct summands and extensions. Thus $\mathscr{F}(A, M, B)$ is a Krull-Schmidt exact category with the canonical exact structure.

Proposition 2.3' Let ${ }_{A} M_{B}$ be a bimodule with ${ }_{A} M$ projective. Then 
(1) $\mathscr{F}(A, M, B)$ has enough injective objects, which are exactly injective $\Lambda$-modules.

(2) $\mathscr{F}(A, M, B)$ has enough projective objects; and the projective objects of $\mathscr{F}(A, M, B)$ are exactly $\left[{ }_{C}^{P}\right]_{\eta_{C, P}^{-1}(\theta)}$ and $\left[\begin{array}{l}0 \\ Q\end{array}\right]$, where $P($ resp. $Q)$ runs over projective $A$-modules (resp. B-modules), and $\theta: C \rightarrow$ $\operatorname{Hom}_{A}(M, P)$ is a projective cover of the left $B$-module $\operatorname{Hom}_{A}(M, P)$. In particular, if in addition $\operatorname{Hom}_{A}(M, A)$ is a projective left $B$-module, then the projective objects of $\mathscr{F}(A, M, B)$ are exactly $\left[\underset{\operatorname{Hom}_{A}(M, P)}{P}\right]_{\varphi}$ and $\left[\begin{array}{l}0 \\ Q\end{array}\right]$.

Corollary 2.4' Let ${ }_{A} M_{B}$ be a bimodule with ${ }_{A} M$ projective. Then $\mathscr{F}(A, M, B)$ is a Frobenius category (with the canonical exact structure) if and only if $A$ and $B$ are selfinjective algebras and $M_{B}$ is projective.

Theorem 1.1' Let $M$ be an A-B-bimodule. Then

(1) The following are equivalent:

(i) ${ }_{A} M$ is projective;

(ii) $\mathscr{F}(A, M, B)$ is a coresolving subcategory of $\Lambda$-mod;

(iii) $L:=\left[\begin{array}{c}A \\ C\end{array}\right]_{\eta_{C, A}^{-1}(\theta)} \oplus\left[\begin{array}{l}0 \\ B\end{array}\right]$ is the unique tilting left $\Lambda$-module, up to multiplicities of indecomposable direct summands, such that $\mathscr{F}(A, M, B)=L^{\perp}$, where $C$ is a projective cover of the $B$-module $\operatorname{Hom}_{A}(M, A)$ with projection $\theta: C \rightarrow \operatorname{Hom}_{A}(M, A)$.

(2) $\mathscr{F}(A, M, B)$ is a covariantly finite subcategory of $\Lambda$-mod. Moreover, if ${ }_{A} M$ is projective, then $\mathscr{F}(A, M, B)$ is a functorially finite subcategory of $\Lambda$-mod, and has Auslander-Reiten sequences.

Corollary 1.2' If ${ }_{A} M$ is projective and $\operatorname{Hom}_{A}(M, A)$ is a projective left $B$-module, then $L=\left[\underset{\operatorname{Hom}_{A}(M, A)}{A}\right]_{\varphi} \oplus$ $\left[\begin{array}{l}0 \\ B\end{array}\right]_{\mathrm{Id}}$ is a cotilting left $\Lambda$-module such that $\mathscr{F}(A, M, B)=L^{\perp}$.

Theorem 1.3. Let ${ }_{A} M_{B}$ be a bimodule such that ${ }_{A} M$ is a projective $A$-module and $\operatorname{Hom}_{A}(M, A)$ is a projective left $B$-module. Then there is a recollement of additive categories

$$
B-\underline{\bmod } \rightleftarrows \mathscr{F}(A, M, B) \rightleftarrows \text { - } \rightleftarrows \text {-mod. }
$$

If in addition $A$ and $B$ are selfinjective algebras, then it is in fact a recollement of singularity categories.

Note that if ${ }_{A} M_{B}$ is an exchangeable bimodule then $\operatorname{Hom}_{A}(M, A)$ is a projective left $B$-module.

4.2. Another description of the epimorphism category induced by a bimodule. Recall that the right module version of $\mathscr{S}(A, M, B)$ is $\mathscr{S}(A, M, B)_{r}$, which is the subcategory of $\bmod \Lambda$ consisting of the triple $(U, V)_{\psi}$, where $U \in \bmod A, V \in \bmod B$, and $\psi: U \otimes_{A} M \rightarrow V$ is a monic $B$-map.

Proposition 4.1. The restriction of $\mathrm{D}: \bmod \Lambda \rightarrow \Lambda-\bmod$ gives a duality $\mathrm{D}: \mathscr{S}(A, M, B)_{r} \rightarrow \mathscr{F}(A, M, B)$.

Proof. For a right $A$-module $U$, denote by $\alpha_{U}$ the adjunction isomorphism

$$
\mathrm{D}\left(U \otimes_{A} M\right)=\operatorname{Hom}_{R}\left(U \otimes_{A} M, J\right) \cong \operatorname{Hom}_{A}\left(M, \operatorname{Hom}_{R}(U, J)\right)=\operatorname{Hom}_{A}(M, \mathrm{D} U)
$$

where $\mathrm{D}=\operatorname{Hom}_{R}(-, J)$ is the duality. For a left $A$-module $X$ and a left $B$-module $Y$, denote by $\eta_{Y, X}$ the adjunction isomorphism $\operatorname{Hom}_{A}\left(M \otimes_{B} Y, X\right) \cong \operatorname{Hom}_{B}\left(Y, \operatorname{Hom}_{A}(M, X)\right)$.

For a right $\Lambda$-module $(U, V)_{\psi}$ with a right $B$-map $\psi: U \otimes_{A} M \rightarrow V$, we have $\mathrm{D} V \stackrel{\mathrm{D}(\psi)}{\rightarrow} \mathrm{D}\left(U \otimes_{A} M\right) \stackrel{\alpha_{U}}{\rightarrow}$ $\operatorname{Hom}_{A}(M, \mathrm{D} U)$, and $\eta_{\mathrm{D} V, \mathrm{D} U}^{-1}: \operatorname{Hom}_{B}\left(\mathrm{D} V, \operatorname{Hom}_{A}(M, \mathrm{D} U)\right) \rightarrow \operatorname{Hom}_{A}\left(M \otimes_{B} \mathrm{D} V, \mathrm{D} U\right)$. Then

$$
\mathrm{D}: \bmod \Lambda \rightarrow \Lambda-\bmod ,(U, V)_{\psi} \mapsto\left[\begin{array}{l}
\mathrm{D} U \\
\mathrm{D} V
\end{array}\right]_{\eta_{\mathrm{D} V, \mathrm{D} U}^{-1}}\left(\alpha_{U} \mathrm{D}(\psi)\right)
$$


with $\eta_{\mathrm{D} V, \mathrm{D} U}^{-1}\left(\alpha_{U} \mathrm{D}(\psi)\right): M \otimes_{B} \mathrm{D} V \rightarrow \mathrm{D} U$. For a left $\Lambda$-module $[\underset{Y}{X}]_{\phi}$ with a left $A$-map $\phi: M \otimes_{B} Y \rightarrow X$, we have $Y \stackrel{\eta_{Y, X}(\phi)}{\rightarrow} \operatorname{Hom}_{A}(M, X) \stackrel{\alpha_{D}^{-1}}{\rightarrow} \mathrm{D}\left(\mathrm{D} X \otimes_{A} M\right)$. Then a quasi-inverse of $\mathrm{D}: \bmod \Lambda \rightarrow \Lambda$-mod is

$$
\mathrm{D}: \Lambda-\bmod \rightarrow \bmod \Lambda, \quad\left[\begin{array}{r}
X \\
Y
\end{array}\right]_{\phi} \mapsto(\mathrm{D} X, \mathrm{D} Y)_{\mathrm{D}\left(\alpha_{\mathrm{D} X}^{-1} \eta_{Y, X}(\phi)\right)}
$$

with $\mathrm{D}\left(\alpha_{\mathrm{D} X}^{-1} \eta_{Y, X}(\phi)\right): \mathrm{D} X \otimes_{A} M \rightarrow \mathrm{D} Y$. In fact,

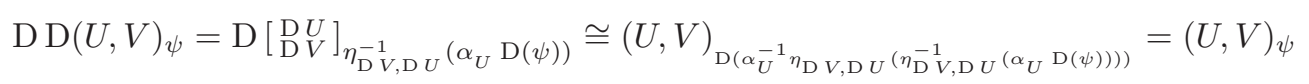

and $\mathrm{DD}\left[\begin{array}{l}X \\ Y\end{array}\right]_{\phi}=\mathrm{D}(\mathrm{D} X, \mathrm{D} Y)_{\mathrm{D}\left(\alpha_{\mathrm{D} X}^{-1} \eta_{Y, X}(\phi)\right)} \cong\left[\begin{array}{l}X \\ Y\end{array}\right]_{\eta_{Y, X}^{-1}\left(\alpha_{\mathrm{D} X} \operatorname{DDD}\left(\alpha_{\mathrm{D}}^{-1} \eta_{Y, X}(\phi)\right)\right)}=\left[\begin{array}{l}X \\ Y\end{array}\right]_{\phi}$.

If a right $\Lambda$-module $(U, V)_{\psi} \in \mathscr{S}(A, M, B)_{r}$, i.e., $\psi: U \otimes_{A} M \rightarrow V$ is a monic right $B$-map, then

$$
\mathrm{D}(U, V)_{\psi}=\left[\begin{array}{l}
\mathrm{D} U \\
\mathrm{D} V
\end{array}\right]_{\eta_{\mathrm{D} V, \mathrm{D} U}^{-1}\left(\alpha_{U} \mathrm{D}(\psi)\right)} \in \mathscr{F}(A, M, B),
$$

since $\eta_{\mathrm{D} V, \mathrm{D} U}\left(\eta_{\mathrm{D} V, \mathrm{D} U}^{-1}\left(\alpha_{U} \mathrm{D}(\psi)\right)\right)=\alpha_{U} \mathrm{D}(\psi): \mathrm{D} V \rightarrow \operatorname{Hom}_{A}(M, \mathrm{D} U)$ is an epic left $B$-map.

If a left $\Lambda$-module $\left[\begin{array}{l}X \\ Y\end{array}\right]_{\phi} \in \mathscr{F}(A, M, B)$, i.e., $\eta_{Y, X}(\phi): Y \rightarrow \operatorname{Hom}_{A}(M, X)$ is an epic left $B$-map, then $\mathrm{D}\left[\begin{array}{l}X \\ Y\end{array}\right]_{\phi}=(\mathrm{D} X, \mathrm{D} Y)_{\mathrm{D}\left(\alpha_{\mathrm{D} X}^{-1} \eta_{Y, X}(\phi)\right)} \in \mathscr{S}(A, M, B)_{r}$, since $\mathrm{D}\left(\alpha_{\mathrm{D} X}^{-1} \eta_{Y, X}(\phi)\right): \mathrm{D} X \otimes_{A} M \rightarrow \mathrm{D} Y$ is monic.

\section{Ringel-Schmidmeier-Simson equivalences via cotilting modules}

Definition 5.1. A Ringel-Schmidmeier-Simson equivalence, in short, an RSS equivalence, induced by a bimodule ${ }_{A} M_{B}$ is an equivalence $F: \mathscr{S}(A, M, B) \cong \mathscr{F}(A, M, B)$ of categories, such that

$$
F\left[\begin{array}{c}
X \\
0
\end{array}\right] \cong\left[\underset{\operatorname{Hom}_{A}(M, X)}{X}\right]_{\varphi}, \forall X \in A \text {-mod, and } F\left[\underset{Y}{M \otimes_{B} Y}\right]_{\mathrm{Id}} \cong\left[\begin{array}{c}
0 \\
Y
\end{array}\right], \forall Y \in B \text {-mod, }
$$

where $\varphi: M \otimes_{B} \operatorname{Hom}_{A}(M, X) \longrightarrow X$ is the involution map.

An RSS equivalence implies a strong symmetry. It was first observed by C. Ringel and M. Schimdmeier [RS2] for ${ }_{A} M_{B}={ }_{A} A_{A}$. For an RSS equivalence induced by a chain without relations we refer to D. Simson [S1-S3], and for RSS equivalences induced by acyclic quivers with monomial relations we refer to [ZX].

5.1. Special cotilting modules induced by exchangeable bimodules. Let $M$ be an $A$ - $B$-bimodule. If $M \otimes_{B} \mathrm{D} B$ is an injective left $A$-module, then by Corollary 1.2, ${ }_{\Lambda} T=\left[\begin{array}{c}\mathrm{D}\left(A_{A}\right) \\ 0\end{array}\right] \oplus\left[\begin{array}{c}M \otimes_{B} \mathrm{D}(B) \\ \mathrm{D}\left(B_{B}\right)\end{array}\right]_{\mathrm{Id}}$ is a cotilting left $\Lambda$-module with $\mathscr{S}(A, M, B)={ }^{\perp} T$. If $\mathrm{D}(A) \otimes_{A} M$ is an injective right $B$-module, then by a right module version of Theorem 1.1 (cf. Subsection 2.4), $U_{\Lambda}=\left(\mathrm{D}\left({ }_{A} A\right), \mathrm{D}\left({ }_{A} A\right) \otimes_{A} M\right)_{\mathrm{Id}} \oplus\left(0, \mathrm{D}\left({ }_{B} B\right)\right)$ is a cotilting right $\Lambda$-module with $\mathscr{S}(A, M, B)_{r}={ }^{\perp}\left(U_{\Lambda}\right)$. If ${ }_{A} M_{B}$ is exchangeable, then both the conditions are satisfied; and by $\mathrm{D}\left({ }_{A} A_{A}\right) \otimes_{A} M \cong M \otimes_{B} \mathrm{D}\left({ }_{B} B_{B}\right)$, we can regard that $T$ and $U$ have the same underlying abelian group $\mathrm{D} A \oplus\left(M \otimes_{B} \mathrm{D} B\right) \oplus \mathrm{D} B$. The following lemma claims that in this case $T$ (and $U$ ) can be endowed with a $\Lambda$ - $\Lambda$-bimodule structure such that $\mathscr{S}(A, M, B)={ }^{\perp}\left({ }_{\Lambda} T\right)$ and $\mathscr{S}(A, M, B)_{r}={ }^{\perp}\left(T_{\Lambda}\right)$ (thus $\left.T_{\Lambda} \cong U_{\Lambda}\right)$.

Lemma 5.2. Let ${ }_{A} M_{B}$ be an exchangeable bimodule with an $A$-B-bimodule isomorphism $g: \mathrm{D}\left({ }_{A} A_{A}\right) \otimes_{A} M \cong$ $M \otimes_{B} \mathrm{D}\left({ }_{B} B_{B}\right)$, and $\Lambda_{\Lambda} T=\left[\begin{array}{c}\mathrm{D} A \\ 0\end{array}\right] \oplus\left[\begin{array}{c}M \otimes_{B} \mathrm{D} B \\ \mathrm{D} B\end{array}\right]_{\mathrm{Id}}$. Then $T$ has a $\Lambda$ - $\Lambda$-bimodule structure such that both ${ }_{\Lambda} T$ and $T_{\Lambda}$ are cotilting modules, $T_{\Lambda} \cong\left(\mathrm{D}\left({ }_{A} A\right), \mathrm{D} A \otimes_{A} M\right)_{\mathrm{Id}} \oplus\left(0, \mathrm{D}\left({ }_{B} B\right)\right)$ as right $\Lambda$-modules, $\mathscr{S}(A, M, B)={ }^{\perp}\left({ }_{\Lambda} T\right)$ and $\mathscr{S}(A, M, B)_{r}={ }^{\perp}\left(T_{\Lambda}\right)$.

Write $T=\left[\begin{array}{cc}\mathrm{D}\left(A_{A}\right) & M \otimes_{B} \mathrm{D}\left(B_{B}\right) \\ 0 & \mathrm{D}\left(B_{B}\right)\end{array}\right]$. Then the right $\Lambda$-action is given by

$$
\left(\begin{array}{cc}
\alpha & m^{\prime} \otimes_{B} \beta \\
0 & \beta^{\prime}
\end{array}\right)\left(\begin{array}{ll}
a & m \\
0 & b
\end{array}\right)=\left(\begin{array}{cc}
\alpha a & g\left(\alpha \otimes_{A} m\right)+m^{\prime} \otimes_{B} \beta b \\
0 & \beta^{\prime} b
\end{array}\right) .
$$


Proof. By Example 1.5(1), ${ }_{A} M_{B}$ satisfies the condition (IP). It follows from Corollary 1.2 that ${ }_{\Lambda} T$ is a cotilting left $\Lambda$-module with $\mathscr{S}(A, M, B)={ }^{\perp}\left({ }_{\Lambda} T\right)$.

Since $g$ is a right $B$-isomorphism, we get a right $\Lambda$-isomorphism

$$
\left(\operatorname{Id}_{\mathrm{D}\left({ }_{A} A\right)}, g^{-1}\right):\left(\mathrm{D}\left({ }_{A} A\right), M \otimes_{B} \mathrm{D} B\right)_{g} \cong\left(\mathrm{D}\left({ }_{A} A\right), \mathrm{D} A \otimes_{A} M\right)_{\mathrm{Id}} .
$$

We endow the abelian group $T=\mathrm{D} A \oplus\left(M \otimes_{B} \mathrm{D} B\right) \oplus \mathrm{D} B$ with a right $\Lambda$-module structure via $g$, i.e., $T_{\Lambda}=\left(\mathrm{D}\left({ }_{A} A\right), M \otimes_{B} \mathrm{D} B\right)_{g} \oplus\left(0, \mathrm{D}\left({ }_{B} B\right)\right)$. Then we get a right $\Lambda$-isomorphism:

$$
T_{\Lambda}=\left(\mathrm{D}\left({ }_{A} A\right), M \otimes_{B} \mathrm{D} B\right)_{g} \oplus\left(0, \mathrm{D}\left({ }_{B} B\right)\right) \cong\left(\mathrm{D}\left({ }_{A} A\right), \mathrm{D} A \otimes_{A} M\right)_{\mathrm{Id}} \oplus\left(0, \mathrm{D}\left({ }_{B} B\right)\right) .
$$

since $g$ is also a left $A$-map, one can easily verify that $T$ is a $\Lambda$ - $\Lambda$-bimodule. We omit the details.

By Example1.5 (1), $\mathrm{D}\left({ }_{A} A_{A}\right) \otimes_{A} M$ is an injective right $B$-module. It follows from the right module version of Theorem 1.1 (cf. Subsection 2.4) that $T_{\Lambda} \cong\left(\mathrm{D}\left({ }_{A} A\right), \mathrm{D} A \otimes_{A} M\right)_{\mathrm{Id}} \oplus\left(0, \mathrm{D}\left({ }_{B} B\right)\right)$ is a cotilting right $\Lambda$-module with $\mathscr{S}(A, M, B)_{r}={ }^{\perp}\left(T_{\Lambda}\right)$.

The following fact will play a crucial role in proving the existence of an RSS equivalence.

Lemma 5.3. Let ${ }_{A} M_{B}$ be an exchangeable bimodule, and ${ }_{\Lambda} T_{\Lambda}$ the $\Lambda$ - $\Lambda$-bimodule $T$ given in Lemma 5.2 . Then there is an algebra isomorphism $\rho: \operatorname{End}_{\Lambda}\left({ }_{\Lambda} T\right)^{o p} \cong \Lambda$, such that under $\rho$, the right module $T_{\operatorname{End}_{\Lambda}\left({ }_{\Lambda} T\right)^{o p}}$ coincides with the right module $T_{\Lambda}$; and there is an algebra isomorphism $\operatorname{End}_{\Lambda}\left(T_{\Lambda}\right) \cong \Lambda$, such that under this algebra isomorphism, the left module $\operatorname{End}_{\Lambda}\left(T_{\Lambda}\right) T$ coincides with the left module $\Lambda_{\Lambda} T$.

Proof. Since $T_{\Lambda}$ is a right $\Lambda$-module, we get the canonical algebra homomorphism $\rho: \Lambda \longrightarrow \operatorname{End}_{\Lambda}\left({ }_{\Lambda} T\right)^{o p}$, $\lambda \mapsto " t \mapsto t \lambda$ ". By Lemma 5.2. $T_{\Lambda}$ is cotilting, so $T_{\Lambda}$ is faithful (Suppose $T \lambda=0$ for $\lambda \in \Lambda$. By a surjective $\Lambda$-map $T_{0} \longrightarrow \mathrm{D} \Lambda$ with $T_{0} \in \operatorname{add} T$, we see $(\mathrm{D} \Lambda) \lambda=0$, i.e., $\mathrm{D}(\lambda \Lambda)=0$. So $\left.\lambda=0\right)$. Thus $\rho$ is an injective map. On the other hand, we have algebra isomorphisms

$$
\begin{aligned}
\operatorname{End}_{\Lambda}(\Lambda T)^{o p} & \cong\left[\begin{array}{cc}
\operatorname{End}_{\Lambda}\left(\left[\begin{array}{c}
\mathrm{D} A \\
0
\end{array}\right]\right) & \operatorname{Hom}_{\Lambda}\left(\left[\begin{array}{c}
M \otimes_{B} \mathrm{D} B \\
\mathrm{D} B
\end{array}\right]_{\mathrm{Id}}\left[\begin{array}{c}
\mathrm{D} A \\
0
\end{array}\right]\right) \\
\operatorname{Hom}_{\Lambda}\left(\left[\begin{array}{c}
\mathrm{D} A \\
0
\end{array}\right],\left[\begin{array}{c}
M \otimes_{B} \mathrm{D} B \\
\mathrm{D} B
\end{array}\right]_{\mathrm{Id}}\right) & \operatorname{End}_{\Lambda}\left(\left[\begin{array}{c}
M \otimes_{B} \mathrm{D} B \\
\mathrm{D} B
\end{array}\right]_{\mathrm{Id}}\right)
\end{array}\right] \\
& \cong\left[\begin{array}{cc}
A^{o p} & 0 \\
M & B^{o p}
\end{array}\right]^{o p} \cong\left[\begin{array}{cc}
A & M \\
0 & B
\end{array}\right]=\Lambda .
\end{aligned}
$$

Denote this algebra isomorphism $\operatorname{End}_{\Lambda}\left({ }_{\Lambda} T\right)^{o p} \cong \Lambda$ by $h$. Since $\Lambda$ is an Artin $R$-algebra, where $R$ is a commutative artinian ring, $h \rho: \Lambda \longrightarrow \Lambda$ is an $R$-endomorphism of artinian $R$-module $\Lambda$. Since $h \rho$ is an injective map, it follows that $h \rho$ is surjective, and hence $\rho$ is surjective (since $h$ is an $R$-module isomorphism).

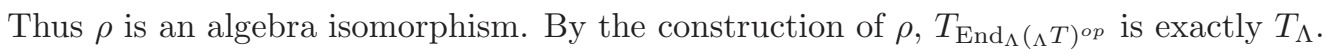

Since $\rho: \operatorname{End}_{\Lambda}\left({ }_{\Lambda} T\right)^{o p} \cong \Lambda$ as algebras, and under $\rho, T_{\operatorname{End}_{\Lambda}\left({ }_{\Lambda} T\right)^{o p}}$ is exactly $T_{\Lambda}$. By the tilting theory, the homomorphism $\Lambda \longrightarrow \operatorname{End}_{\Lambda}\left(T_{\Lambda}\right)$ given by $\lambda \mapsto " t \mapsto \lambda t$ " is an algebra isomorphism ([HR, p.409]), and hence $\operatorname{End}_{\Lambda}\left(T_{\Lambda}\right) T$ is exactly ${ }_{\Lambda} T$ (one can also prove this by the same argument as above).

5.2. Existence of RSS equivalences. For any left $\Gamma$-module $L$, following $[\mathrm{AR}]$, let $\mathcal{X}_{\Gamma} L$ be the subcategory of $\Gamma$-mod consisting of $\Gamma$-modules ${ }_{\Gamma} X$ such that there is an exact sequence

$$
0 \longrightarrow X \longrightarrow L_{0} \stackrel{f_{0}}{\longrightarrow} L_{1} \longrightarrow \cdots \longrightarrow L_{j} \stackrel{f_{j}}{\longrightarrow} L_{j+1} \longrightarrow \cdots
$$

with $L_{j} \in \operatorname{add}\left({ }_{\Gamma} L\right)$ and $\left.\operatorname{Im} f_{j} \in{ }^{\perp}{ }_{\Gamma} L\right)$ for $j \geq 0$. The following fact is in T. Wakamatsu [W, Prop. 1]. 
Lemma 5.4. ([W]) For any $\Gamma$-module $\Gamma_{\Gamma} L$ with $C:=\operatorname{End}_{\Gamma}(L)^{o p}$, we have a contravariant functor

$$
\operatorname{Hom}_{\Gamma}\left(-{ }_{\Gamma} L\right): \mathcal{X}_{\Gamma} L \longrightarrow{ }^{\perp}\left(L_{C}\right)
$$

such that if $\Gamma_{\Gamma} X \in \mathcal{X}_{\Gamma} L$, then the canonical $\Gamma$-map ${ }_{\Gamma} X \rightarrow \operatorname{Hom}_{C}\left(\operatorname{Hom}_{\Gamma}\left(\Gamma_{\Gamma} X, \Gamma_{\Gamma} L\right), L_{C}\right)$ is an isomorphism.

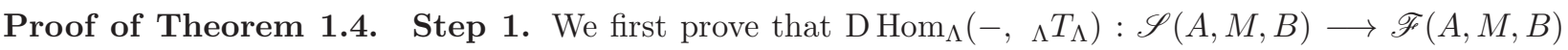
is an equivalence of categories. By Proposition 4.1. $\mathrm{D}: \mathscr{S}(A, M, B)_{r} \longrightarrow \mathscr{F}(A, M, B)$ is a duality. So, it suffices to prove that $\operatorname{Hom}_{\Lambda}\left(-,{ }_{\Lambda} T_{\Lambda}\right): \mathscr{S}(A, M, B) \longrightarrow \mathscr{S}(A, M, B)_{r}$ is a duality.

By Lemma 5.2 $T$ is a $\Lambda$ - $\Lambda$-bimodule such that $\mathscr{S}(A, M, B)={ }^{\perp}\left({ }_{\Lambda} T\right)$ and $\mathscr{S}(A, M, B)_{r}={ }^{\perp}\left(T_{\Lambda}\right)$. Since ${ }_{\Lambda} T$ is cotilting, it follows from M. Auslander and I. Reiten [AR, Thm. 5.4(b)] that $\mathcal{X}_{\Lambda} T={ }^{\perp}\left({ }_{\Lambda} T\right)$. Thus $\mathscr{S}(A, M, B)={ }^{\perp}\left({ }_{\Lambda} T\right)=\mathcal{X}_{\Lambda} T$.

By Lemma 5.3, there is an algebra isomorphism $\rho: \operatorname{End}_{\Lambda}\left({ }_{\Lambda} T\right)^{o p} \cong \Lambda$, such that $T_{\operatorname{End}_{\Lambda}\left({ }_{\Lambda} T\right)^{o p}}=T_{\Lambda}$ under $\rho$. So we can apply Lemma 5.4 to ${ }_{\Lambda} T$ to get a contravariant functor:

$$
\operatorname{Hom}_{\Lambda}\left(-,{ }_{\Lambda} T\right): \mathcal{X}_{\Lambda} T=\mathscr{S}(A, M, B) \longrightarrow \mathscr{S}(A, M, B)_{r}={ }^{\perp}\left(T_{\Lambda}\right)
$$

such that for $X \in \mathscr{S}(A, M, B)=\mathcal{X}_{\Lambda}{ }_{T}$, the canonical left $\Lambda$-map ${ }_{\Lambda} X \longrightarrow \operatorname{Hom}_{\Lambda}\left(\operatorname{Hom}_{\Lambda}\left({ }_{\Lambda} X,{ }_{\Lambda} T\right), T_{\Lambda}\right)$ is an isomorphism.

Similarly, $T_{\Lambda}$ is a cotilting module and $\mathscr{S}(A, M, B)_{r}={ }^{\perp}\left(T_{\Lambda}\right)=\mathcal{X}_{T_{\Lambda}}$ ([AR, Thm. 5.4(b)]). By Lemma 5.3 there is an algebra isomorphism $\operatorname{End}_{\Lambda}\left(T_{\Lambda}\right) \cong \Lambda$, such that $\operatorname{End}_{\Lambda}\left(T_{\Lambda}\right) T={ }_{\Lambda} T$ under this isomorphism. So we can apply the right module version of Lemma 5.4 to $T_{\Lambda}$ to get a contravariant functor

$$
\operatorname{Hom}_{\Lambda}\left(-, T_{\Lambda}\right): \mathcal{X}_{T_{\Lambda}}=\mathscr{S}(A, M, B)_{r} \longrightarrow \mathscr{S}(A, M, B)={ }^{\perp}\left({ }_{\Lambda} T\right)
$$

such that for each $Y \in \mathscr{S}(A, M, B)_{r}=\mathcal{X}_{T_{\Lambda}}$, the canonical left $\Lambda$-map $Y_{\Lambda} \longrightarrow \operatorname{Hom}_{\Lambda}\left(\operatorname{Hom}_{\Lambda}\left(Y_{\Lambda}, T_{\Lambda}\right),{ }_{\Lambda} T\right)$ is an isomorphism. Thus $\operatorname{Hom}_{\Lambda}\left(-,{ }_{\Lambda} T\right): \mathscr{S}(A, M, B) \longrightarrow \mathscr{S}(A, M, B)_{r}$ is a duality with a quasi-inverse $\operatorname{Hom}_{\Lambda}\left(-, T_{\Lambda}\right): \mathscr{S}(A, M, B)_{r} \longrightarrow \mathscr{S}(A, M, B)$.

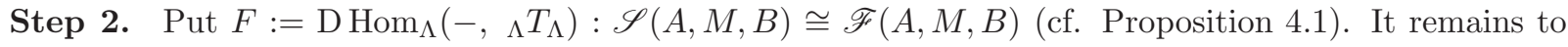
prove $F\left(\left[\begin{array}{c}X \\ 0\end{array}\right]\right) \cong\left[\underset{\operatorname{Hom}_{A}(M, X)}{X}\right]_{\varphi}$ and $F\left(\left[\begin{array}{c}M \otimes_{B} Y \\ Y\end{array}\right]_{\mathrm{Id}}\right) \cong\left[\begin{array}{l}0 \\ Y\end{array}\right]$ for $X \in A$-mod and $Y \in B$-mod. This is true by direct computations, which are included as an Appendix at the end of this paper.

5.3. RSS equivalences and the Nakayama functors. It is natural to ask when the Nakayama functor

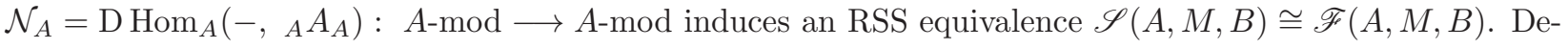
note by $\mathcal{G P}(\Lambda)($ resp. $\mathcal{G I}(\Lambda))$ the subcategory of $\Lambda$-mod consisting of Gorenstein-projective (resp. Gorensteininjective) modules ([EJ]).

Proposition 5.5. Let ${ }_{A} M_{B}$ be an exchangeable $A$-B-bimodule. Then the restriction of the Nakayama functor $\mathcal{N}_{\Lambda}$ gives an $\operatorname{RSS}$ equivalence $\mathscr{S}(A, M, B) \cong \mathscr{F}(A, M, B)$ if and only if both $A$ and $B$ are Frobenius algebras. If this is the case, we have $\mathscr{S}(A, M, B)=\mathcal{G P}(\Lambda)$ and $\mathscr{F}(A, M, B)=\mathcal{G I}(\Lambda)$.

Proof. If the restriction of $\mathcal{N}_{\Lambda}$ gives an RSS equivalence, then we have $\mathcal{N}_{\Lambda}\left[\begin{array}{c}A \\ 0\end{array}\right]=\left[\begin{array}{c}{ }^{A} A \\ \operatorname{Hom}_{A}(M, A)\end{array}\right]_{\operatorname{Id}}$ and $\mathcal{N}_{\Lambda}\left[\begin{array}{c}M \\ B\end{array}\right]_{\text {Id }}=$ $\left[\begin{array}{c}0 \\ B B\end{array}\right]$. However

$$
\mathcal{N}_{\Lambda}\left[\begin{array}{c}
A \\
0
\end{array}\right]=\operatorname{DHom}_{\Lambda}\left(\left[\begin{array}{c}
A \\
0
\end{array}\right], \Lambda\right)=\operatorname{DHom}_{\Lambda}\left(\left[\begin{array}{c}
A \\
0
\end{array}\right],\left[\begin{array}{c}
A \\
0
\end{array}\right] \oplus\left[\begin{array}{c}
M \\
B
\end{array}\right]_{\mathrm{Id}}\right)=\mathrm{D}(A, M)_{\mathrm{Id}}=\left[\begin{array}{c}
\mathrm{D}\left(A_{A}\right) \\
\mathrm{D} M
\end{array}\right]_{\mathrm{Id}},
$$


and

$$
\mathcal{N}_{\Lambda}\left[\begin{array}{c}
M \\
B
\end{array}\right]_{\mathrm{Id}}=\mathrm{DH}_{\operatorname{Hom}}\left(\left[\left[_{B}^{M}\right]_{\mathrm{Id}}, \Lambda\right)=\mathrm{DHom}_{\Lambda}\left(\left[\begin{array}{c}
M \\
B
\end{array}\right]_{\mathrm{Id}},\left[\begin{array}{c}
A \\
0
\end{array}\right] \oplus\left[\begin{array}{c}
M \\
B
\end{array}\right]_{\mathrm{Id}}\right)=\mathrm{D}(0, B)=\left[\begin{array}{c}
0 \\
\mathrm{D}\left(B_{B}\right)
\end{array}\right] .\right.
$$

So $\mathrm{D}\left(A_{A}\right) \cong{ }_{A} A$ and $\mathrm{D}\left(B_{B}\right) \cong{ }_{B} B$, i.e., $A$ and $B$ are Frobenius algebras.

Conversely, if both $A$ and $B$ are Frobenius algebras, then replacing ${ }_{\Lambda} T_{\Lambda}$ by ${ }_{\Lambda} \Lambda_{\Lambda}$ and using the same arguments as in the proofs of Lemmas 5.2 and 5.3 and Theorem 1.4 we get an RSS equivalence

$$
\mathcal{N}_{\Lambda}=\mathrm{D}_{\operatorname{Hom}}\left(-,{ }_{\Lambda} \Lambda_{\Lambda}\right): \mathscr{S}(A, M, B) \longrightarrow \mathscr{F}(A, M, B)
$$

If both $A$ and $B$ are Frobenius algebras and both ${ }_{A} M$ and $M_{B}$ are projective, then $\Lambda$ is a Gorenstein algebra (cf. [Z2, Lemma 2.1]), and then by [Z2, Thm. 2.2] and its dual, $\mathscr{S}(A, M, B)=\mathcal{G P}(\Lambda)$ and $\mathscr{F}(A, M, B)=$ $\mathcal{G I}(\Lambda)$. This completes the proof.

Remark. For any algebra $\Gamma$, the Nakayama functor always gives an equivalence $\mathcal{N}_{\Gamma}: \mathcal{G P}(\Gamma) \cong \mathcal{G I}(\Gamma)$ (see [Bel, Prop. 3.4]). Also, if $A$ and $B$ are Frobenius, then there is a left $A$-isomorphism $f_{1}: \mathrm{D}\left(A_{A}\right) \cong{ }_{A} A$ and a left $B$-isomorphism $g_{1}: \mathrm{D}\left(B_{B}\right) \cong{ }_{B} B$, so ${ }_{\Lambda} T=\left[\begin{array}{c}\mathrm{D}\left(A_{A}\right) \\ 0\end{array}\right] \oplus\left[\begin{array}{c}M \otimes_{B} \mathrm{D}\left(B_{B}\right) \\ \mathrm{D}\left(B_{B}\right)\end{array}\right]_{\mathrm{Id}} \cong\left[\begin{array}{c}A_{A} A \\ 0\end{array}\right] \oplus\left[\begin{array}{c}A_{B} M \\ B\end{array}\right]_{\mathrm{Id}}={ }_{\Lambda} \Lambda$. By the symmetry a Frobenius algebra, there is a right $A$-isomorphism $f_{2}: \mathrm{D}\left({ }_{A} A\right) \cong A_{A}$ and a right $B$-isomorphism $g_{2}: \mathrm{D}\left({ }_{B} B\right) \cong B_{B}$. So $T_{\Lambda} \cong\left(\mathrm{D}\left({ }_{A} A\right), \mathrm{D}\left({ }_{A} A\right) \otimes_{A} M\right)_{\operatorname{Id}} \oplus\left(0, \mathrm{D}\left({ }_{B} B\right)\right) \cong\left(A_{A}, M_{B}\right)_{\mathrm{Id}} \oplus\left(0, B_{B}\right)=\Lambda_{\Lambda}$. But $T ¥ \Lambda$ as $\Lambda$ - $\Lambda$-modules in general, since a Frobenius algebra is not necessarily a symmetric algebra. Thus, the "if part" of Proposition 5.5 is not a corollary of Theorem 1.4

5.4. We illustrate Theorem 1.4 and Proposition 5.5. The conjunction of paths of a quiver is from the right to the left.

Example 5.6. Let $B$ be the path algebra $k(b \longrightarrow a)$. We write the indecomposable $B$-modules as ${ }_{1}^{0}=S(a)=$ $P(a),{ }_{1}^{1}=P(b)=I(a),{ }_{0}^{1}=S(b)=I(b)$. Let $A:=B \oplus B$ and ${ }_{A} M_{B}:={ }_{A} A_{B}$. So ${ }_{A} M_{B}$ is an exchangeable bimodule, and $\Lambda:=\left[\begin{array}{cc}A & M \\ 0 & B\end{array}\right]=\left[\begin{array}{ccc}B & 0 & B \\ 0 & B & B \\ 0 & 0 & B\end{array}\right]=B \otimes_{k} k Q$, where $Q$ is the quiver $1 \stackrel{\alpha}{\longleftarrow} 3 \stackrel{\beta}{\longrightarrow} 2$. Thus $\Lambda$ is given by the quiver

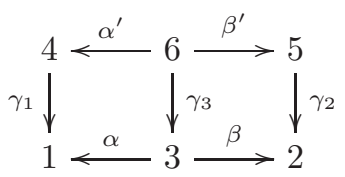

with relations $\gamma_{1} \alpha^{\prime}-\alpha \gamma_{3}, \quad \beta \gamma_{3}-\gamma_{2} \beta^{\prime}$. We will write a $\Lambda$-module as a representation of $Q$ over algebra $B$ (see e.g. [ZX], [LZ]). Thus a $\Lambda$-module is written as $X_{1} \stackrel{X_{\alpha}}{\longleftarrow} X_{3} \stackrel{X_{\beta}}{\longrightarrow} X_{2}$, where $X_{1}, X_{2}, X_{3} \in B$-mod, and $X_{\alpha}$ and $X_{\beta}$ are B-maps. For example, the indecomposable projective $\Lambda$-module $P(6)=\begin{array}{lll}1 & 1 & 1\end{array} 1$ at vertex 6 is

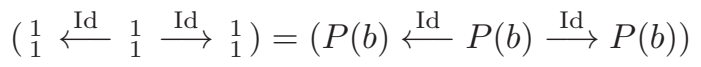


With this notation, the Auslander-Reiten quiver of $\Lambda$ is

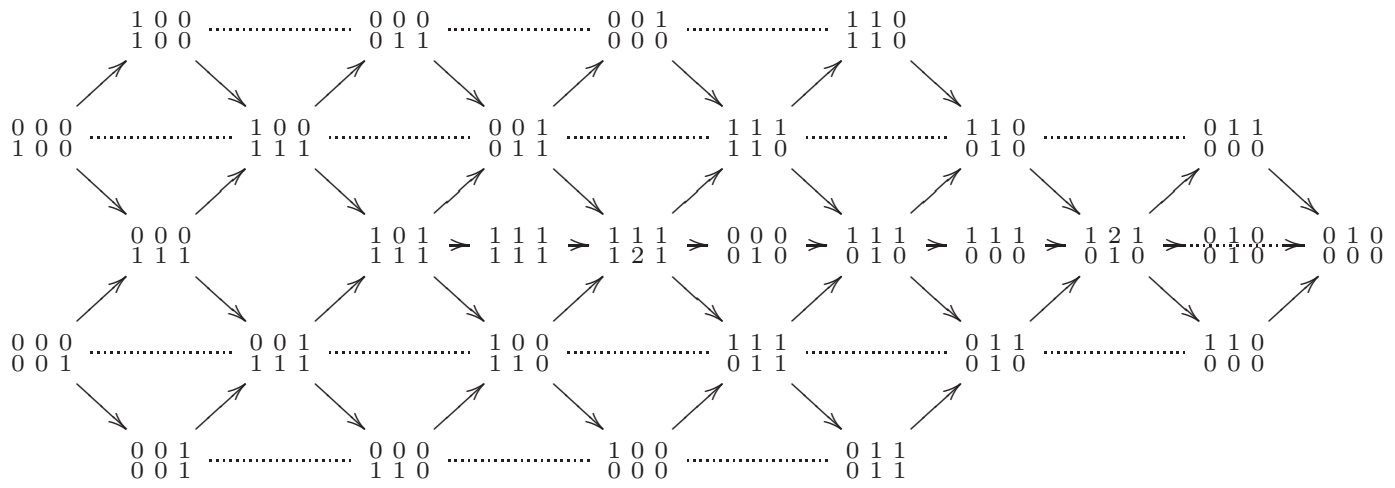

Since $\Lambda$ is of the form $\left[\begin{array}{cc}A & M \\ 0 & B\end{array}\right]$, a $\Lambda$-module $X_{1} \stackrel{X_{\alpha}}{\longleftarrow} X_{3} \stackrel{X_{\beta}}{\longrightarrow} X_{2}$ is also written as a triple $\left[\begin{array}{c}X_{1} \oplus X_{2} \\ X_{3}\end{array}\right]_{\phi}$, where $X_{1} \oplus X_{2} \in A$-mod and $\phi: M \otimes_{B} X_{3} \rightarrow X_{1} \oplus X_{2}$ is exactly the A-map $\left(\begin{array}{cc}X_{\alpha} & 0 \\ 0 & X_{\beta}\end{array}\right): X_{3} \oplus X_{3} \rightarrow X_{1} \oplus X_{2}$. Thus it is in $\mathscr{S}(A, M, B)$ if and only if $X_{\alpha}$ and $X_{\beta}$ are monic. So the Auslander-Reiten quiver of $\mathscr{S}(A, M, B)$ is:

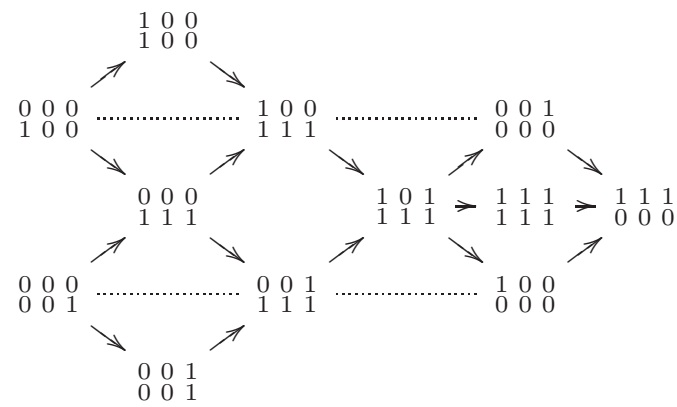

Note that $\left(X_{2} \stackrel{X_{\alpha}}{\rightarrow} X_{1} \stackrel{X_{\beta}}{\longleftarrow} X_{3}\right)=\left[\begin{array}{c}X_{1} \oplus X_{2} \\ X_{3}\end{array}\right]_{\phi} \in \mathscr{F}(A, M, B)$ if and only if $\left(\begin{array}{l}X_{\alpha} \\ X_{\beta}\end{array}\right): X_{3} \longrightarrow X_{1} \oplus X_{2}$ is an epic B-map (in particular, $X_{\alpha}$ and $X_{\beta}$ are epic; but this is not sufficient). So the Auslander-Reiten quiver of $\mathscr{F}(A, M, B)$ is:

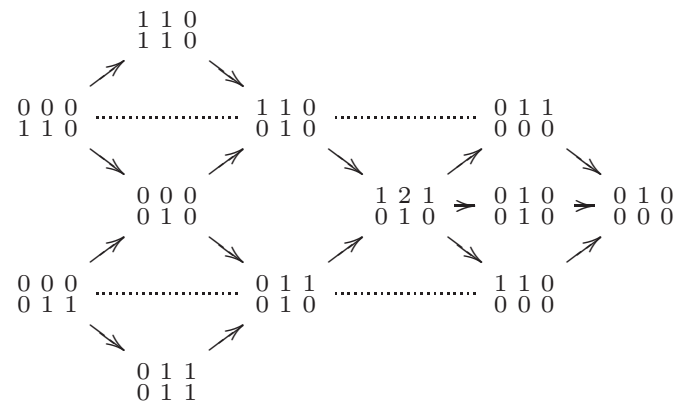

There is a unique RSS equivalence, sending an indecomposable object in $\mathscr{S}(A, M, B)$ to the one in $\mathscr{F}(A, M, B)$, in the same positions of the Auslander-Reiten quivers. Note that this RSS equivalence is not given by the Nakayama functor $\mathcal{N}_{\Lambda}$, since it does not send projective $\Lambda$-modules to injective $\Lambda$-modules.

Example 5.7. Let $\Lambda$ be the algebra given by the quiver $2 \stackrel{\beta}{\longrightarrow} 1 \bigcirc$ with relation $\alpha^{2}$. Then $\Lambda:=\left[\begin{array}{ll}A & M \\ 0 & B\end{array}\right]$, where $A:=k[\alpha] /\left\langle\alpha^{2}\right\rangle, B:=e_{2} \Lambda e_{2} \cong k$, and ${ }_{A} M_{k}=e_{1} \Lambda e_{2}=k \beta \oplus k \alpha \beta \cong{ }_{A} A_{k}$. Then ${ }_{A} M_{k}$ is an exchangeable 
bimodule. The Auslander-Reiten quiver of $\Lambda$ is

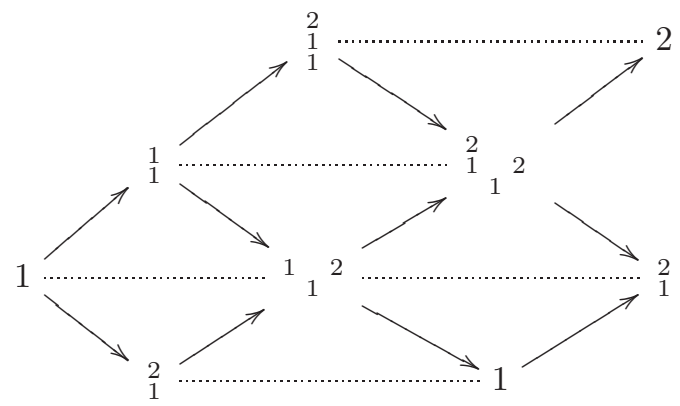

where the two 1 's represents the same module, and the two ${ }_{1}^{2}$ 's also represents the same module. To compute $\mathscr{S}(A, M, B)$ and $\mathscr{F}(A, M, B)$, we need to write a $\Lambda$-module $N$ as the form $\left[\begin{array}{l}e_{1} N \\ e_{2} N\end{array}\right]_{\phi}$, where $\phi: M \otimes_{k} e_{2} N \longrightarrow$ $e_{1} N$ is the A-map given by the $\Lambda$-actions:

$$
\beta \otimes_{k} e_{2} n \mapsto \beta e_{2} n, \quad \alpha \beta \otimes_{k} e_{2} n \mapsto \alpha \beta e_{2} n, \quad \forall n \in N
$$

For example, the $\Lambda$-module ${ }_{1}^{2}{ }_{2}$ has a basis $e_{1}, \alpha, u, v$ with the $\Lambda$-actions

\begin{tabular}{|l|l|l|l|l|}
\hline & $e_{1}$ & $\alpha$ & $u$ & $v$ \\
\hline$e_{1}$ & $e_{1}$ & $\alpha$ & 0 & 0 \\
\hline$e_{2}$ & 0 & 0 & $u$ & $v$ \\
\hline$\alpha$ & $\alpha$ & 0 & 0 & 0 \\
\hline$\beta$ & 0 & 0 & $\alpha$ & $e_{1}$ \\
\hline$\alpha \beta$ & 0 & 0 & 0 & $\alpha$ \\
\hline
\end{tabular}

it follows that ${ }_{1}^{2}{ }_{1}^{2}=\left[\begin{array}{c}k e_{1} \oplus k \alpha \\ k u \oplus k v\end{array}\right]_{\phi}$ with the $A$-map $\phi$ given by the $\Lambda$-actions:

$$
\beta \otimes_{k} u \mapsto \beta u=\alpha, \quad \beta \otimes_{k} v \mapsto \beta v=e_{1}, \quad \alpha \beta \otimes_{k} u \mapsto \alpha \beta u=0, \quad \alpha \beta \otimes_{k} v \mapsto \alpha \beta v=\alpha
$$

So $\phi$ is not monic and hence ${ }_{1}^{2}{ }_{1}^{2} \notin \mathscr{S}(A, M, B)$. Also, since the $\Lambda$-module ${ }_{1}{ }^{2}$ has a basis $e_{1}$, $\alpha, u$ with the $\Lambda$-actions given by the table above, it follows that ${ }^{1}{ }_{1}^{2}=\left[\begin{array}{c}k e_{1} \oplus k \alpha \\ k u\end{array}\right]_{\phi}$ with the A-map $\phi$ given as above. So $\phi$ is not monic and hence ${ }^{1}{ }_{1}^{2} \notin \mathscr{S}(A, M, B)$. In this way we see that $\mathscr{S}(A, M, B)$ has 3 indecomposable objects: $1, \frac{1}{1}, \frac{1}{1}$.

Similarly a $\Lambda$-module $N=\left[\begin{array}{c}e_{1} N \\ e_{2} N\end{array}\right]_{\phi} \in \mathscr{F}(A, M, B)$ if and only if $\varphi: e_{2} N \longrightarrow \operatorname{Hom}_{A}\left(M, e_{1} N\right)=e_{1} N$ is epic, where $\varphi$ is the image of $\phi: M \otimes_{k} e_{2} N \longrightarrow e_{1} N$ under the adjunction isomorphism. Note that $\varphi: e_{2} N \longrightarrow e_{1} N$ is exactly given by the actions of $\beta$. For example, since the $\Lambda$-module ${ }_{1}^{2}$ has a basis $\alpha, u$ with the $\Lambda$-actions given by the table above, it follows that ${ }_{1}^{2}=\left[\begin{array}{l}k \alpha \\ k u\end{array}\right]_{\phi}$ with $\varphi: k u \longrightarrow k \alpha$ given by $u \mapsto \beta u=\alpha . \quad$ So $_{1}^{2} \in \mathscr{F}(A, M, B)$. In this way we see that $\mathscr{F}(A, M, B)$ has 3 indecomposable objects: $\stackrel{2}{1},{ }_{1}^{2} 2,2$.

There is a unique RSS equivalence given by $1 \mapsto{ }_{1}^{2}, \quad{ }_{1}^{1} \mapsto{ }_{1}^{2},{ }_{1}^{2} \mapsto 2$. Note that $\mathscr{S}(A, M, B)=\mathcal{G P}(\Lambda)$ and $\mathscr{F}(A, M, B)=\mathcal{G I}(\Lambda)$, and this RSS equivalence is given by the Nakayama functor $\mathcal{N}_{\Lambda}$. 
5.5. The following examples show that if ${ }_{A} M_{B}$ is not exchangeable, then the existence of an RSS equivalence can not be guaranteed. They also show that $\mathscr{S}(A, M, B)$ is not the separated monomorphism category of the corresponding quiver in the sense of $[\mathrm{ZX}]$, in general.

Example 5.8. (1) Let $A$ be the path algebra $k(2 \longrightarrow 1), B:=k$, and ${ }_{A} M_{k}:={ }_{A}\left(A e_{1}\right)_{k}=01$. Then $\Lambda:=\left[\begin{array}{cc}A & M \\ 0 & k\end{array}\right]=\left[\begin{array}{lll}k & k & k \\ 0 & k & 0 \\ 0 & 0 & k\end{array}\right]$ is just the path algebra $k Q$, where $Q$ is the quiver $2 \longrightarrow 1 \longleftarrow 3$. The AuslanderReiten quiver of $\Lambda$-mod is

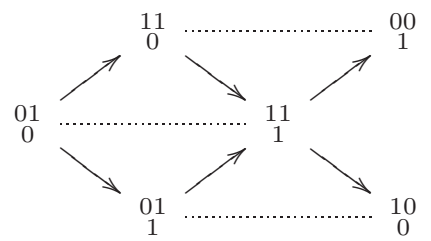

where we denote a $\Lambda$-module $V_{2} \longrightarrow V_{1} \longleftarrow V_{3}$ by $\frac{\operatorname{dim}_{k} V_{2} \operatorname{dim}_{k} V_{1}}{\operatorname{dim}_{k} V_{3}}$. Since

$$
\mathrm{D}\left({ }_{A} A_{A}\right) \otimes_{A} M=\mathrm{D}\left({ }_{A} A_{A}\right) \otimes_{A} A e_{1} \cong \mathrm{D}\left(e_{1} A\right) \not A e_{1}=M \otimes_{B} \mathrm{D}\left({ }_{B} B_{B}\right)
$$

${ }_{A} M_{k}$ is not an exchangeable bimodule. In fact, ${ }_{A} M_{k}$ also does not satisfy the condition (IP). The monomorphism category $\mathscr{S}(A, M, k)$ induced by ${ }_{A} M_{k}$ is

$$
\mathscr{S}(A, M, k)=\left\{\left[\begin{array}{c}
X \\
Y
\end{array}\right]_{\phi} \in \Lambda-\bmod \mid X \in A-\bmod , Y \in k-\bmod , M \otimes_{k} Y \stackrel{\phi}{\hookrightarrow} X \text { is a monic A-map }\right\} .
$$

Thus $\mathscr{S}(A, M, k)$ has 5 indecomposable objects $\underset{0}{01}, \underset{0}{11}, \underset{1}{01}, \underset{1}{11}, \underset{0}{10}$. While the epimorphism category $\mathscr{F}(A, M, k)$ induced by ${ }_{A} M_{k}$ is

$$
\begin{aligned}
\mathscr{F}(A, M, k) & =\left\{\left[\begin{array}{c}
X \\
Y
\end{array}\right]_{\phi} \in \Lambda-\bmod \mid X \in A-\bmod , Y \in k-\bmod , Y \stackrel{\varphi}{\rightarrow} \operatorname{Hom}_{A}(M, X) \text { is an epick-map }\right\} \\
& =\left\{\left[\begin{array}{c}
X \\
Y
\end{array}\right]_{\phi} \in \Lambda-\bmod \mid X \in A-\bmod , Y \in k-\bmod , Y \stackrel{\varphi}{\rightarrow} e_{1} X \text { is an epic k-map }\right\}
\end{aligned}
$$

where $\varphi:=\eta_{X, Y}(\phi)$ and $\eta_{X, Y}: \operatorname{Hom}_{A}\left(M \otimes_{k} Y, X\right) \cong \operatorname{Hom}_{k}\left(Y, \operatorname{Hom}_{A}(M, X)\right)$ is the adjunction isomorphism. So $\mathscr{F}(A, M, k)$ has only 4 indecomposable objects $\underset{1}{01}, \underset{1}{11}, \underset{1}{00}, \underset{0}{10}$. Thus $\mathscr{S}(A, M, k) \nsubseteq \mathscr{F}(A, M, k)$.

Note that the indecomposable objects of the separated monomorphism category $\operatorname{smon}(Q, 0, k)$ are exactly the indecomposable projective $\Lambda$-modules. See [ZX, Exam. 2.3]. So $\mathscr{S}(A, M, k) \nsucceq \operatorname{smon}(Q, 0, k)$.

(2) Let $A$ and $B:=k$ be as in (1), and ${ }_{A} M_{k}:=S(2)=10$. Since ${ }_{A} M$ is not projective, ${ }_{A} M_{k}$ is not exchangeable (note that $\mathrm{D}\left({ }_{A} A_{A}\right) \otimes_{A} M=\mathrm{D}\left({ }_{A} A_{A}\right) \otimes_{A} S(2)=\mathrm{D}\left({ }_{A} A_{A}\right) \otimes_{A} e_{2} S(2) \cong \mathrm{D}\left(e_{2} A\right)=S(2) \cong M \otimes{ }_{B}$ $\mathrm{D}\left({ }_{B} B_{B}\right)$; and ${ }_{A} M_{k}$ satisfies the condition $\left.(\mathrm{IP})\right)$. Then $\Lambda:=\left[\begin{array}{cc}A & M \\ 0 & k\end{array}\right]=\left[\begin{array}{ccc}k & k & 0 \\ 0 & k & k \\ 0 & 0 & k\end{array}\right] \cong k Q / I$ with $Q=3 \stackrel{\beta}{\longrightarrow} 2 \stackrel{\alpha}{\longrightarrow} 1$ and $I=\langle\alpha \beta\rangle$. The Auslander-Reiten quiver of $\Lambda$-mod is

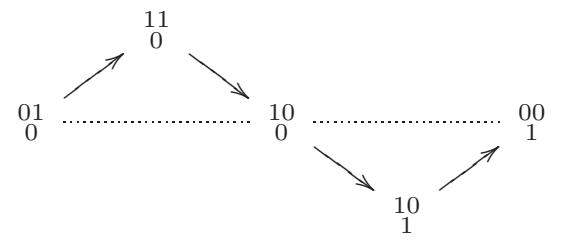

where a $\Lambda$-module $V_{3} \longrightarrow V_{2} \longrightarrow V_{1}$ is denoted by $\operatorname{dim}_{k} V_{2} \operatorname{dim}_{k} V_{1} V_{3}$. The monomorphism category $\mathscr{S}(A, M, k)=$ $\left\{\left[\begin{array}{c}X \\ Y\end{array}\right]_{\phi} \in \Lambda\right.$-mod $\mid M \otimes_{k} Y \stackrel{\phi}{\hookrightarrow} X$ is a monic A-map $\}$ has 4-indecomposable objects $\begin{gathered}01 \\ 0\end{gathered}, \begin{gathered}11 \\ 0\end{gathered}, \begin{gathered}10 \\ 0\end{gathered}, \quad \begin{gathered}10 \\ 1\end{gathered}$ The epimorphism category $\mathscr{F}(A, M, k)=\left\{\left[\begin{array}{c}X \\ Y\end{array}\right]_{\phi} \in \Lambda-\bmod \mid Y \stackrel{\varphi}{\rightarrow} \operatorname{Hom}_{A}(S(2), X)\right.$ is an epic k-map $\}$ has also 4 
indecomposable objects $\underset{0}{01}, \underset{0}{11},{ }_{1}^{10},{ }_{1}^{00}$. We claim that there are no RSS equivalences $F: \mathscr{S}(A, M, k) \cong$ $\mathscr{F}(A, M, k)$. Otherwise, by the definition of an RSS equivalence we get a contradiction

$$
0 \neq \operatorname{Hom}_{\Lambda}\left(\begin{array}{c}
11 \\
0
\end{array}, \begin{array}{c}
10 \\
1
\end{array}\right) \cong \operatorname{Hom}_{\Lambda}\left(F\left(\begin{array}{c}
11 \\
0
\end{array}\right), F\left(\begin{array}{c}
10 \\
1
\end{array}\right)\right)=\operatorname{Hom}_{\Lambda}\left(\begin{array}{c}
11 \\
0
\end{array}, \begin{array}{c}
00 \\
1
\end{array}\right)=0
$$

By [ZX, Exam. 2.3], the indecomposable objects of the separated monomorphism category $\operatorname{smon}(Q, I, k)$ are exactly the indecomposable projective $\Lambda$-modules. So $\mathscr{S}(A, M, k) \varsubsetneqq \operatorname{smon}(Q, 0, k)$.

We propose the following problems.

1. What is a sufficient and necessary condition for the existence of an RSS equivalence?

2. Whether or not an RSS equivalence is unique?

\section{Appendix: computations of Step 2 in the proof of Theorem 1.4}

Put $F=\operatorname{DHom}_{\Lambda}(-, T): \mathscr{S}(A, M, B) \cong \mathscr{F}(A, M, B)$. Let $X \in A$-mod and $Y \in B$-mod. To prove $F\left(\left[\begin{array}{l}X \\ 0\end{array}\right]\right) \cong\left[\begin{array}{c}X \\ \operatorname{Hom}_{A}(M, X)\end{array}\right]_{\varphi}$ and $F\left(\left[\begin{array}{c}M \otimes_{B} Y \\ Y\end{array}\right]_{\mathrm{Id}}\right) \cong\left[\begin{array}{c}0 \\ Y\end{array}\right]$, where $\varphi: M \otimes_{B} \operatorname{Hom}_{A}(M, X) \longrightarrow X$ is the involution map, by the proof of Proposition 4.1 it suffices to prove

$$
\operatorname{Hom}_{\Lambda}\left(\left[\begin{array}{l}
X \\
0
\end{array}\right], T\right) \cong\left(\mathrm{D} X, \mathrm{D}_{\operatorname{Hom}}(M, X)\right)_{\mathrm{D}\left(\alpha_{\mathrm{D} X}^{-1}\right)}
$$

where $\alpha_{\mathrm{D} X}: \mathrm{D}(\mathrm{D} X \otimes M) \cong \operatorname{Hom}_{A}(M, X)$ is the adjunction isomorphism, $\mathrm{D}\left(\alpha_{\mathrm{D} X}^{-1}\right): \mathrm{D} X \otimes_{A} M \longrightarrow$

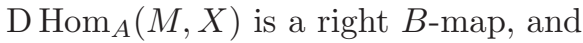

$$
\operatorname{Hom}_{\Lambda}\left(\left[\underset{Y}{M \otimes_{B} Y}\right]_{\mathrm{Id}}, T\right) \cong(0, \mathrm{D} Y)
$$

Put $e_{1}:=\left(\begin{array}{ll}1 & 0 \\ 0 & 0\end{array}\right), e_{2}:=\left(\begin{array}{ll}0 & 0 \\ 0 & 1\end{array}\right)$. Then as left $\Lambda$-modules we have $T e_{1}=\left[\begin{array}{c}\mathrm{D}\left(A_{A}\right) \\ 0\end{array}\right]$ and $T e_{2}=\left[\begin{array}{c}M \otimes_{B} \mathrm{D} B \\ \mathrm{D} B\end{array}\right]_{\mathrm{Id}} \cong$ $\left[\begin{array}{c}\mathrm{D}(A) \otimes_{A} M \\ \mathrm{D}\left(B_{B}\right)\end{array}\right]_{g^{-1}}$. For any left $\Lambda$-module $L, \operatorname{Hom}_{\Lambda}(L, T)$ is a right $\Lambda$-module. Then $\operatorname{Hom}_{\Lambda}(L, T) e_{1} \cong \operatorname{Hom}_{\Lambda}\left(L, T e_{1}\right)$ is a right $A$-module and $\operatorname{Hom}_{\Lambda}(L, T) e_{2} \cong \operatorname{Hom}_{\Lambda}\left(L, T e_{2}\right)$ is a right $B$-module, in the obvious way, and we have a right $\Lambda$-isomorphism

$$
\operatorname{Hom}_{\Lambda}(L, T) \cong\left(\operatorname{Hom}_{\Lambda}\left(L, T e_{1}\right), \operatorname{Hom}_{\Lambda}\left(L, T e_{2}\right)\right)_{\phi}
$$

where $\phi$ is explicit given by:

$$
\phi: \operatorname{Hom}_{\Lambda}\left(L, T e_{1}\right) \otimes_{A} M \longrightarrow \operatorname{Hom}_{\Lambda}\left(L, T e_{2}\right), \quad f \otimes_{A} m \mapsto " l \mapsto f(l)\left(\begin{array}{cc}
0 & m \\
0 & 0
\end{array}\right) e_{2} " .
$$

Applying (6.3) to $L=\left[\begin{array}{c}M \otimes_{B} Y \\ Y\end{array}\right]_{\mathrm{Id}}$ we get right $\Lambda$-isomorphisms:

$$
\begin{aligned}
\operatorname{Hom}_{\Lambda}\left(\left[\begin{array}{c}
M \otimes_{B} Y \\
Y
\end{array}\right]_{\mathrm{Id}}, T\right) & =\left(\operatorname{Hom}_{\Lambda}\left(\left[\begin{array}{c}
M \otimes_{B} Y \\
Y
\end{array}\right]_{\mathrm{Id}}, T e_{1}\right), \operatorname{Hom}_{\Lambda}\left(\left[\begin{array}{c}
M \otimes_{B} Y \\
Y
\end{array}\right]_{\mathrm{Id}}, T e_{2}\right)\right) \\
& =\left(\operatorname{Hom}_{\Lambda}\left(\left[\begin{array}{c}
M \otimes_{B} Y \\
Y
\end{array}\right]_{\mathrm{Id}},\left[\begin{array}{c}
\mathrm{D}\left(A_{A}\right) \\
0
\end{array}\right]\right), \operatorname{Hom}_{\Lambda}\left(\left[\begin{array}{c}
M \otimes_{B} Y \\
Y
\end{array}\right]_{\mathrm{Id}},\left[\begin{array}{c}
M \otimes_{B} \mathrm{D}\left(B_{B}\right) \\
\mathrm{D}\left(B_{B}\right)
\end{array}\right]_{\mathrm{Id}}\right)\right. \\
& \cong\left(0, \operatorname{Hom}_{B}\left(Y, \mathrm{D}\left(B_{B}\right)\right) \cong(0, \mathrm{D} Y) .\right.
\end{aligned}
$$

This proves (6.2). 
Applying (6.3) to $L=\left[\begin{array}{c}X \\ 0\end{array}\right]$ we get right $\Lambda$-isomorphisms:

$$
\begin{aligned}
\operatorname{Hom}_{\Lambda}\left(\left[\begin{array}{l}
X \\
0
\end{array}\right], T\right) & =\left(\operatorname{Hom}_{\Lambda}\left(\left[\begin{array}{c}
X \\
0
\end{array}\right], T e_{1}\right), \operatorname{Hom}_{\Lambda}\left(\left[\begin{array}{c}
X \\
0
\end{array}\right], T e_{2}\right)\right) \\
& =\left(\operatorname{Hom}_{\Lambda}\left(\left[\begin{array}{c}
X \\
0
\end{array}\right],\left[\begin{array}{c}
\mathrm{D}\left(A_{A}\right) \\
0
\end{array}\right]\right), \operatorname{Hom}_{\Lambda}\left(\left[\begin{array}{c}
X \\
0
\end{array}\right],\left[\begin{array}{c}
M \otimes_{B} \mathrm{D}\left(B_{B}\right) \\
\mathrm{D}\left(B_{B}\right)
\end{array}\right]_{\mathrm{Id}}\right)\right) \\
& \cong\left(\mathrm{D} X, \operatorname{Hom}_{\Lambda}\left(\left[\begin{array}{c}
X \\
0
\end{array}\right],\left[\begin{array}{c}
\mathrm{D}(A) \otimes M \\
\mathrm{D}\left(B_{B}\right)
\end{array}\right]_{g^{-1}}\right)\right) \\
& \cong\left(\mathrm{D} X, \operatorname{Hom}_{A}\left(X, \mathrm{D}(A) \otimes_{A} M\right)\right)_{\phi}
\end{aligned}
$$

and by (6.4), $\phi$ is explicitly given by

$$
\left.\phi: \mathrm{D} X \otimes_{A} M \longrightarrow \operatorname{Hom}_{A}\left(X, \mathrm{D}(A) \otimes_{A} M\right)\right), \quad \alpha \otimes_{A} m \mapsto “ x \mapsto \alpha_{x} \otimes_{A} m "
$$

where $\alpha_{x} \in \mathrm{D} A$ sends $a$ to $\alpha(a x)$.

To prove (6.1), it is clear that we need a right $B$-isomorphism $\psi: \mathrm{D} \mathrm{Hom}_{A}(M, X) \cong \operatorname{Hom}_{A}\left(X, \mathrm{D}(A) \otimes_{A} M\right)$. For this, we need to use the assumption that ${ }_{A} M$ is projective. Without loss of the generality, one can take ${ }_{A} M=A e$ for some idempotent element $e \in A$. First, we have group isomorphisms

$$
\mathrm{D}_{\operatorname{Hom}}(M, X)=\mathrm{D}_{H^{\prime}}(A e, X) \cong \mathrm{D}(X) e \cong \operatorname{Hom}_{A}(X, \mathrm{D}(e A)) \cong \operatorname{Hom}_{A}\left(X, \mathrm{D}(A) \otimes_{A} M\right) \text {. }
$$

This isomorphism $\psi: \mathrm{D} \mathrm{Hom}_{A}(M, X) \cong \operatorname{Hom}_{A}\left(X, \mathrm{D}(A) \otimes_{A} M\right)$ of abelian groups is explicitly given by

$$
\gamma \mapsto " x \mapsto \gamma_{x} \otimes_{A} e^{"}
$$

where $\gamma_{x} \in \mathrm{D} A$ sends $a \in A$ to $\gamma\left(f_{a, x}\right)$, and $f_{a, x} \in \operatorname{Hom}_{A}(M, X)$ sends $m=c e \in M=A e$ to $\max$. We claim that $\psi$ is a right $B$-map, and hence $\psi$ is a right $B$-module isomorphism.

In fact, for each $b \in B$, suppose $e b=v_{b} e \in M=A e$ for some $v_{b} \in A$. Then for each $x \in X$ we have

$$
\psi(\gamma b)(x)=(\gamma b)_{x} \otimes e, \text { with }(\gamma b)_{x} \in \mathrm{D} A
$$

and

$$
(\psi(\gamma) b)(x)=\psi(\gamma)(x) b=\gamma_{x} \otimes_{A} e b=\gamma_{x} \otimes_{A} v_{b} e=\gamma_{x} v_{b} e \otimes_{A} e, \text { with } \gamma_{x} v_{b} \in \mathrm{D} A
$$

Thus, it suffices to show $(\gamma b)_{x}(a)=\left(\gamma_{x} v_{b} e\right)(a)=\gamma_{x}\left(v_{b} e a\right)$ for each $a \in A$, i.e., $(\gamma b)\left(f_{a, x}\right)=\gamma\left(f_{v_{b} e a, x}\right)$. That is $\gamma\left(b f_{a, x}\right)=\gamma\left(f_{v_{b} e a, x}\right)$. This is really true, since both $b f_{a, x}$ and $f_{v_{b} e a, x}$ sends $m$ to

$$
\left(b f_{a, x}\right)(m)=f_{a, x}(m b)=\operatorname{mbax}=c e(e b) a x=c e\left(v_{b} e\right) a x=\operatorname{mv} v_{b} e a x=f_{v_{b} e a, x}(m)
$$

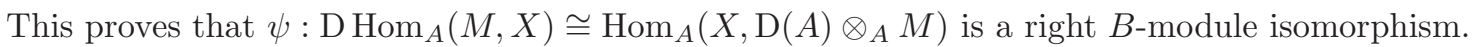

So, we get the right $\Lambda$-isomorphism

$$
\left(\operatorname{Id}_{\mathrm{D} X}, \psi^{-1}\right):\left(\mathrm{D} X, \operatorname{Hom}_{A}\left(X, \mathrm{D}(A) \otimes_{A} M\right)\right)_{\psi \mathrm{D}\left(\alpha_{\mathrm{D} X}^{-1}\right)} \cong\left(\mathrm{D} X, \mathrm{D} \mathrm{Hom}_{A}(M, X)\right)_{\mathrm{D}\left(\alpha_{\mathrm{D} X}^{-1}\right)},
$$

where $\alpha_{\mathrm{D} X}: \mathrm{D}(\mathrm{D} X \otimes M) \cong \operatorname{Hom}_{A}(M, X)$ sends $\beta \in \mathrm{D}(\mathrm{D} X \otimes M)$ to $f \in \operatorname{Hom}_{A}(M, X)$ such that

$$
\beta\left(\alpha \otimes_{A} m\right)=\alpha f(m), \forall \alpha \in \mathrm{D} X, m \in M,
$$

$\alpha_{\mathrm{D} X}^{-1}: \operatorname{Hom}_{A}(M, X) \longrightarrow \mathrm{D}\left(\mathrm{D} X \otimes_{A} M\right)$ is given by $f \mapsto " \beta: \alpha \otimes_{A} m \mapsto \alpha f(m) "$, and $\mathrm{D}\left(\alpha_{\mathrm{D} X}^{-1}\right): \mathrm{D} X \otimes_{A} M \longrightarrow$ $\mathrm{D} \operatorname{Hom}_{A}(M, X)$ is given by

$$
\alpha \otimes_{A} m \mapsto " \delta: f \mapsto \alpha f(m) "
$$




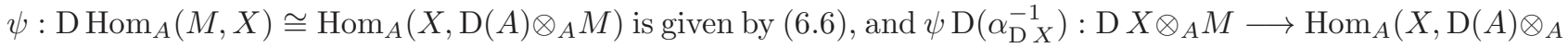
$M)$. To prove (6.1), it suffices to prove $\phi=\psi \mathrm{D}\left(\alpha_{\mathrm{D} X}^{-1}\right)$. Thus by (6.6) we have

$$
\left(\psi \mathrm{D}\left(\alpha_{\mathrm{D} X}^{-1}\right)\left(\alpha \otimes_{A} m\right)\right)(x)=\psi(\delta)(x)=\delta_{x} \otimes_{A} e
$$

where $\delta_{x} \in \mathrm{D} A$ sends $a \in A$ to $\delta\left(f_{a, x}\right)=\alpha f_{a, x}(m)=\alpha(\max )$. Comparing with (6.5) we see $\delta_{x}=\alpha_{x} c e=$ $\alpha_{x} m$, since $\alpha_{x} m(a)=\alpha_{x}(m a)=\alpha(\max )=\delta_{x}(a)$. It follows that

$$
\left(\psi \mathrm{D}\left(\alpha_{\mathrm{D} X}^{-1}\right)\left(\alpha \otimes_{A} m\right)\right)(x)=\delta_{x} \otimes_{A} e=\alpha_{x} c e \otimes_{A} e=\alpha_{x} \otimes_{A} m=\phi\left(\alpha \otimes_{A} m\right)(x) .
$$

This proves $\psi \mathrm{D}\left(\alpha_{\mathrm{D} X}^{-1}\right)=\phi$, and hence completes the proof.

\section{REFERENCES}

[ASS] I. Assem, D. Simson, A. Skowroński, Elements of the representation theory of associative algebras, Vol.1. Techniques of representation theory, Lond. Math. Soc. Students Texts 65, Cambridge University Press, 2006.

[AR] M. Auslander, I. Reiten, Applications of contravariantly finite subcategories, Adv. Math. 86(1991), 111-152.

[ARS] M. Auslander, I. Reiten, S. O. Smalø, Representation Theory of Artin Algebras, Cambridge Studies in Adv. Math. 36., Cambridge Univ. Press, 1995.

[AS] M. Auslander, S. O. Smalø, Almost split sequences in subcategories, J. Algebra 69(1981), 426-454.

[BBD] A. A. Beilinson, J. Bernstein and P. Deligne, Faisceaux Perves, Asterique 100 (Soc. Math., France, 1982).

[Bel] A. Beligiannis, Cohen-Macaulay modules, (co)torsion pairs and virtually Gorenstein algebras, J. Algebra 288(1)(2005), 137-211.

[Bir] G. Birkhoff, Subgroups of abelian groups, Proc. Lond. Math. Soc. II, Ser. 38(1934), 385-401.

[Buch] R.-O. Buchweitz, Maximal Cohen-Macaulay modules and Tate cohomology over Gorenstein rings, Unpublished manuscript, Hamburg (1987), 155pp.

[C] X. W. Chen, The stable monomorphism category of a Frobenius category, Math. Res. Lett. 18(1)(2011), $125-137$.

[CZ] X. W. Chen, P. Zhang, Quotient triangulated categories, Manuscripta Mathematica, 123 (2007), 167-183.

[EJ] E. E. Enochs, O. M. G. Jenda, Relative homological algebra, De Gruyter Exp. Math. 30. Walter De Gruyter Co., 2000.

[FZ] J. Feng, P. Zhang, Types of Serre subcategories of Grothendieck categories, J. Algebra (to appear)

[FP] V. Franjou, T. Pirashvili, Comparison of abelian categories recollement, Doc. Math. 9(2004), 41-56.

[H1] D. Happel, Triangulated categories in representation theory of finite dimensional algebras, London Math. Soc. Lecture Notes Ser. 119, Cambridge Uni. Press, 1988.

[H2] D. Happel, Reduction techniques for homological conjectures, Tsukuba J. Math. 17(1)(1993), 115-130.

[HR] D. Happle, C. M. Ringel, Tilted algebras, Trans. Amer. Math. Soc. 274(2)(1982), 399-443.

[IKM] O. Iyama, K. Kato, J.-I. Miyachi, Recollement on homotopy categories and CohenCMacaulay modules, J. K-Theory $8(3)(2011), 507-541$.

[Ke] B. Keller, Derived categories and universal problems, Comm. Algebra 19(1991), 699-747.

[Kö] S. König, Tilting complexes, perpendicular categories and recollements of derived module categories of rings, J. Pure Appl. Algebra 73(1991), 211-232.

[KS] H. Krause, Ø. Solberg, Applications of cotorsion pairs, J. London Math. Soc.(2)68(3)(2003), 631-650.

[Ku] N. J. Kuhn, Generic representation theory of the finite general linear groups and the steenrod algebra: II, K-Theory 8(1994), 395-428.

[KLM1] D. Kussin, H. Lenzing, H. Meltzer, Nilpotent operators and weighted projective lines, J. Reine Angew. Math. 685(6)(2010), 33-71.

[KLM2] D. Kussin, H. Lenzing, H. Meltzer, Triangle singularities, ADE-chains, and weighted projective lines, Adv. Math. 237(2013), 194-251.

[LZ] X. H. Luo, P. Zhang, Separated monic representations I: Gorenstein-projective modules, J. Algebra 479(2017), 1-34.

[MV] R. MacPherson, K. Vilonen, Elementary construction of perverse sheaves, Invent. Math. 84(1986), 403-436.

[N] A. Neeman, Triangulated categories, Ann. Math. Studies 148, Princeton University Press, Princeton, NJ, 2001. 
[O] D. Orlov, Triangulated categories of singularities and D-branes in Landau-Ginzburg models, Proc. Steklov Inst. Math. 246(3)(2004), 227-248.

[PS] B. J. Parshall, L. L. Scott, Derived categories, quasi-hereditary algebras and algebric groups, Math. Lecture Notes Series 3, Carleton Univ. (1988), 1-105.

[PV] C. Psaroudakis, J. Vitoria, Recollement of module categories, Appl. Categ. Structures 22(2014), 579-593.

[R] C. M. Ringel, Tame algebras and integral quadratic forms, Lecture Notes in Math. 1099, Springer-Verlag, 1984.

[RS1] C. M. Ringel, M. Schmidmeier, Submodules categories of wild representation type, J. Pure Appl. Algebra 205(2)(2006), $412-422$.

[RS2] C. M. Ringel, M. Schmidmeier, The Auslander-Reiten translation in submodule categories, Trans. Amer. Math. Soc. 360(2)(2008), 691-716.

[RS3] C. M. Ringel, M. Schmidmeier, Invariant subspaces of nilpotent operators I, J. rein angew. Math. 614(2008), 1-52.

[S1] D. Simson, Representation types of the category of subprojective representations of a finite poset over $K[t] /\left(t^{m}\right)$ and a solution of a Birkhoff type problem, J. Algebra 311(2007), 1-30.

[S2] D. Simson, Tame-wild dichotomy of Birkhoff type problems for nilpotent linear operators, J. Algebra 424(2015), $254-293$.

[S3] D. Simson, Representation-finite Birkhoff type problems for nilpotent linear operators, to appear in: JPAA

[W] T. Wakamatsu, On modules with trivial self-extensions, J. Algebra 114(1988), 106-114.

[XZ] B. L. Xiong, P. Zhang, Gorenstein-projective modules over triangular matrix Artin algebras, J. Algebra Appl. 11(4)(2012), 1250066.

[Z1] P. Zhang, Monomorphism categories, cotilting theory, and Gorenstein-projective modules, J. Algebra 339(2011), $181-202$.

[Z2] P. Zhang, Gorenstein-projective modules and symmetric recollements, J. Algebra 388(2013), 65-80.

[ZX] P. Zhang, B. L. Xiong, Separated monic representations II: Frobenius subcategories and RSS equivalences, arXiv:1707.04866 1 [math.RT]

Bao-Lin Xiong Email: xiongbaolin@gmail.com

Department of Mathematics, Beijing University of Chemical Technology, Beijing 100029, P. R. China

$\mathrm{Pu}$ Zhang Email: pzhang@sjtu.edu.cn

School of Mathematics, Shanghai Jiao Tong University, Shanghai 200240, P. R. China

Yue-Hui Zhang Email: zyh@sjtu.edu.cn

School of Mathematics, Shanghai Jiao Tong University, Shanghai 200240, P. R. China 